\title{
Common microscopic structural origin for water's thermodynamic and dynamic anomalies
}

Rui Shi, John Russo, and Hajime Tanaka

Citation: J. Chem. Phys. 149, 224502 (2018); doi: 10.1063/1.5055908

View online: https://doi.org/10.1063/1.5055908

View Table of Contents: http://aip.scitation.org/toc/jcp/149/22

Published by the American Institute of Physics

\section{Articles you may be interested in}

Perspective: Excess-entropy scaling

The Journal of Chemical Physics 149, 210901 (2018); 10.1063/1.5055064

Improved general-purpose five-point model for water: TIP5P/2018

The Journal of Chemical Physics 149, 224507 (2018); 10.1063/1.5070137

Perspective: Crossing the Widom line in no man's land: Experiments, simulations, and the location of the liquid-liquid critical point in supercooled water

The Journal of Chemical Physics 149, 140901 (2018); 10.1063/1.5046687

Calculation of the water-octanol partition coefficient of cholesterol for SPC, TIP3P, and TIP4P water The Journal of Chemical Physics 149, 224501 (2018); 10.1063/1.5054056

High and low density patches in simulated liquid water

The Journal of Chemical Physics 149, 204507 (2018); 10.1063/1.5053559

Perspective: Computational modeling of accurate cellular membranes with molecular resolution

The Journal of Chemical Physics 149, 220901 (2018); 10.1063/1.5055007

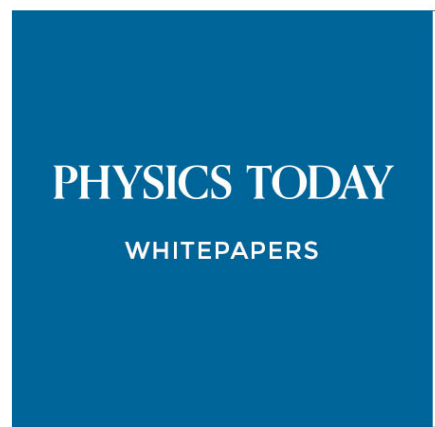

ADVANCED LIGHT CURE ADHESIVES

Take a closer look at what these environmentally friendly adhesive systems can do
READ NOW

PRESENTED BY

QMASTERBOND 


\title{
Common microscopic structural origin for water's thermodynamic and dynamic anomalies
}

\author{
Rui Shi, ${ }^{1}$ John Russo, ${ }^{1,2}$ and Hajime Tanaka ${ }^{1, a)}$ \\ ${ }^{1}$ Department of Fundamental Engineering, Institute of Industrial Science, University of Tokyo, \\ 4-6-1 Komaba, Meguro-ku, Tokyo 153-8505, Japan \\ ${ }^{2}$ School of Mathematics, University of Bristol, Bristol BS8 1TW, United Kingdom
}

(Received 12 September 2018; accepted 9 November 2018; published online 11 December 2018)

\begin{abstract}
Water displays a vast array of unique properties, known as water's anomalies, whose origin remains subject to hot debate. Our aim in this article is to provide a unified microscopic physical picture of water's anomalies in terms of locally favored structures, encompassing both thermodynamic and dynamic anomalies, which are often attributed to different origins. We first identify locally favored structures via a microscopic structural descriptor that measures local translational order and provide direct evidence that they have a hierarchical impact on the anomalies. At each state point, the strength of thermodynamic anomalies is directly proportional to the amount of locally favored structures, while the dynamic properties of each molecule depend on the local structure surrounding both itself and its nearest neighbors. To incorporate this, we develop a novel hierarchical two-state model. We show by extensive simulations of two popular water models that both thermodynamic and kinetic anomalies can be almost perfectly explained by the temperature and pressure dependence of these local and non-local versions of the same structural descriptor, respectively. Moreover, our scenario makes three unique predictions in supercooled water, setting it apart from other scenarios: (1) Presence of an "Arrhenius-to-Arrhenius" crossover upon cooling, as the origin of the apparent "fragile-to-strong" transition; (2) maximum of dynamic heterogeneity around $20 \mathrm{~K}$ below the Widom line and far above the glass transition; (3) violation of the Stokes-Einstein-Debye relation at $\sim 2 T_{\mathrm{g}}$, rather than $1.2 T_{\mathrm{g}}$ typical of normal glass-formers. These predictions are verified by recent measurement of water's diffusion at very low temperatures (point 1) and discoveries from our extensive simulations (points 2-3). We suggest that the same scenario may generally apply to water-like anomalies in liquids tending to form locally favored structures, including not only other important tetrahedral liquids such as silicon, germanium, and silica, but also metallic and chalcogenide liquids. Published by AIP Publishing. https://doi.org/10.1063/1.5055908
\end{abstract}

\section{INTRODUCTION}

Understanding the anomalous behavior of water has attracted considerable effort, and several scenarios have been proposed to explain its unique properties. ${ }^{1-6}$ Among these, the second critical point scenario is widely considered to be the most comprehensive description of water's behavior at low temperatures. ${ }^{2,7}$ It invokes the existence of the so-called Widom line ${ }^{4,5,8,9}$ linked to a second critical point, which acts as a line of finite yet maximum critical fluctuations responsible for the rapid increase of thermodynamic response functions upon cooling. ${ }^{10-13}$ This model explains thermodynamic anomalies by power-law divergences.

The dynamical properties of water also undergo rapid change in the region where thermodynamic properties change anomalously. ${ }^{14-16}$ All the hallmarks of glassy behavior (an increase in dynamic heterogeneity, breakdown of the Stokes-Einstein-Debye relation, etc.) are observed. These dynamic anomalies are often described also by power-law

a)Electronic mail: tanaka@iis.u-tokyo.ac.jp divergences, ${ }^{1,3,5}$ originated from either a glass transition [more precisely, mode-coupling theory ${ }^{17-20}$ (MCT)] or a thermodynamic (limit of mechanical stability) singularity. However, no divergence has ever been detected by measurements in supercooled, amorphous, and confined water, and instead a "fragileto-strong" (power-law to Arrhenius) transition is observed, which is further rationalized as a consequence of crossing the Widom line. ${ }^{8,9,21-28}$

In a recent study, ${ }^{29}$ we have provided an alternative explanation of water's dynamic anomalies. According to this scenario, the observed fragile-to-strong transition is actually a crossover between two Arrhenius regimes, each corresponding to a different state (type of local structures) of water: a high-density $\rho$ state at high temperatures and a low-density $S$ state at low temperatures. The dynamical transition should then be described as an Arrhenius-to-Arrhenius transition, which coincides with the change in the composition of local structures of water at different state points. The work in Ref. 29 also showed that this scenario also naturally explains phenomena usually attributed to an underlying glass transition (such as the breakdown of the Stokes-Einstein-Debye relation) and made some additional predictions (such as the maximization of 
heterogeneities) that were verified in simulations. In this article, we expand on the work in Ref. 29, providing a more detailed analysis of both thermodynamic and dynamic anomalies of water. In particular, we present a unique hierarchical two-state model that quantitatively captures the emergence of both classes of anomalies in water. It shows how thermodynamic anomalies are determined directly from the amount of locally favored structures, while dynamic anomalies are determined by the local environment of each molecule. This hierarchical structure of the model predicts the existence of two characteristic lines, which we call static and dynamic Schottky (DS) lines: a static line, along which (noncritical) static fluctuations are maximized, and a dynamic line, where dynamic fluctuations are maximized. This last point allows us to observe for the first time a unique behavior in water, i.e., a maximum in the dynamical heterogeneities and in the dynamical correlation length as a function of temperature.

Two-state models have been applied successfully in the past to explain the thermodynamic anomalies of real and simulated water by many authors. ${ }^{4,30-42}$ Previously, we also showed that a two-state scenario can explain real water's dynamic anomalies (more precisely, viscosity anomaly) in a temperature range of $258 \mathrm{~K}-373 \mathrm{~K} \cdot{ }^{32-34}$ Recently, by accurate viscosity measurements, Caupin and co-workers ${ }^{40,42}$ also reported a good fitting of the two-state model to water's dynamic properties. However, all these studies were based on a phenomenological two-state description of various physical quantities and lacked microscopic support: in other words, the basic two states were unidentified at a molecular level and their presence was assumed. Attempts to construct a twostate model on the basis of microscopic structural information were made in Refs. 38 and 43 by using a structural parameter $r_{5}$ and in Refs. 38 and 44 by using the local structural index, ${ }^{45}$ both of which only consider the distance of oxygen neighbor(s) from the central one. In 2014, we developed a microscopic two-state model that is capable to describe the thermodynamic anomalies. ${ }^{46}$ In this work, we introduced a new structural descriptor $\zeta$ to quantify the degree of translational order in the second shell (see below on its details). ${ }^{46}$ By taking into account hydrogen bond (H-bond) formation, $\zeta$ largely improves the detection of locally favored structures and thus provides a solid microscopic structural basis for a twostate description of liquid water. ${ }^{47}$ However, the applicability of the two-state model to dynamic anomalies in a deeply supercooled regime, where different scenarios may possibly be distinguished, is still not clear. In particular, direct pieces of evidence supporting the two-state scenario at the molecular level are still missing. Furthermore, it is not clear how dynamic heterogeneity and the breakdown of the Stokes-Einstein-Debye relation, which have been regarded as key features supporting the glass phenomenology glass-transition-based scenario, can be explained by the two-state scenario. Thus the validity of the two-state scenario for dynamic anomalies still remains elusive.

In this article, we examine computer simulations of two popular models of water, the TIP5P and ST2 water models, and build from the ground-up a two-state description based entirely on microscopic information. This will be used to quantitatively predict the location and intensity of the anomalies (as a function of both temperature and pressure) and will be shown to be in excellent agreement with our simulation results and previous experimental observations. We also discover a maximum of dynamic heterogeneity around $20 \mathrm{~K}$ below the Widom line and far above the glass transition and show that it is a distinctive two-state feature that is hard to explain within any other scenarios.

\section{THEORETICAL BACKGROUND}

\section{A. Two-state model}

Here we briefly explain the theoretical framework of a two-state model. In this model, water is treated as a dynamical mixture of two states and its free energy can be expressed as follows: ${ }^{32-38,46,48}$

$$
G=G_{\rho}+s \Delta G+k_{\mathrm{B}} T[s \ln s+(1-s) \ln (1-s)]+J s(1-s),
$$

where $s$ is the fraction of the $S$ state, $\Delta G=G_{S}-G_{\rho}, G_{S}$ and $G_{\rho}$ are the free energies of pure $S$ and $\rho$ states, respectively, $J$ is the cooperativity, describing the energy gain if two states phase-separate, $k_{\mathrm{B}}$ is the Boltzmann's constant, and $T$ is the temperature. A possible entropic origin of the cooperativity was also suggested. ${ }^{35}$ The two states can transform between each other on a short time scale, and equilibrium can be reached if

$$
\frac{\partial G}{\partial s}=\Delta G+k_{\mathrm{B}} T \ln \left(\frac{s}{1-s}\right)+J(1-2 s)=0 .
$$

If there is no critical point, or if it exists but the temperature and pressure are far from the critical region, we can assume the cooperativity $J$ to be negligible ${ }^{32-34}$ (see Sec. II B). Furthermore, the free energy difference $\Delta G$ can be decomposed into energy $(\Delta E)$, entropy $(\Delta \sigma)$, and volume $(\Delta V)$ contributions as $\Delta G=\Delta E-T \Delta \sigma+P \Delta V$. Therefore, the fraction of the $S$ state, $s$, can be described by

$$
s=\frac{1}{1+\exp \left(\frac{\Delta E-T \Delta \sigma+P \Delta V}{k_{\mathrm{B}} T}\right)} .
$$

For example, the volume anomaly is given by $V=\left(V_{\rho}\right.$ $+s \Delta V) / n$, where $\Delta V=V_{S}-V_{\rho}$ is the molar volume difference of $S$ and $\rho$ states and $n$ is the average number of molecules involved in each state, which converts the two-state description on the state basis to the simulation/experimental description on the molecular basis. In our two-state model, the less ordered $\rho$ state shows normal temperature and pressure dependence, like regular liquids. For TIP5P and ST2 water (see below), we found that the temperature dependence of $\rho$ state behaviors can be well described by a linear function, whereas a slightly bent quadratic function best describes the pressure dependence. Hereafter, the values of $\Delta E$ are expressed in units of temperature $(\mathrm{K})$ by dividing them by $k_{\mathrm{B}}$.

\section{B. Cooperativity}

In the above two-state model, cooperativity $J$ defines the energy gain upon mixing of the two states. If $J>0$, cooperative 
formation of the $S$ state is energetically favored and the model predicts a gas-liquid-like critical point for the order parameter $s$ at $T_{\mathrm{c}}=J /\left(2 k_{\mathrm{B}}\right)$, below which the two states separate into two phases under constant volume. ${ }^{32,33,46,49,50}$ This critical point is naturally linked to the proposed second critical point at which a first-order phase transition line between two forms of water ends. So our model predicts the presence of a second critical point. However, since the critical point (217 K and 3400 bars for TIP5P water ${ }^{51}$ ) is located far from the region where anomalies are considered in this study, we can safely ignore the $J$ term in this version of the two-state model. We will show more evidence confirming the weakness of cooperativity in Secs. IV B 6 and IV E 3.

\section{SIMULATION METHODS}

Molecular dynamics simulations were carried out on a system of 1000 TIP5P water molecules in a cubic box with periodic boundary conditions by using the GROMACS package (version 4.6.5). ${ }^{52}$ Intermolecular interactions were cut off at $9 \AA .{ }^{53}$ The NPT ensemble was employed in all the simulations, with temperature and pressure kept constant by using the Berendsen thermostat and barostat, respectively. A time step of $1 \mathrm{fs}$ was used for all the simulations. Equilibration was checked by comparing the diffusion coefficients calculated from the first and last half of each trajectory. A total of $78 \mu$ s well-equilibrated trajectories were sampled for statistical analyses.

As we mentioned above in the Introduction, different scenarios for water's anomalies predict different behaviors only in the deeply supercooled regime, far below the Widom line $T_{\mathrm{W}}$. In this work, extensive simulations were performed for a wide range of temperatures from $430 \mathrm{~K}$ to $226 \mathrm{~K}$ (far below $T_{\mathrm{W}}=255.5 \mathrm{~K}$ for TIP5P water at $1 \mathrm{bar}$ ). Table I lists the total length of the molecular dynamics simulations (after equilibration) and the reorientational relaxation time of TIP5P water at 1 bar. Except for the lowest two temperatures, all the simulation times (after equilibration) were longer than the reorientational time by at least two orders of magnitude. In order to further improve the statistics, three, ten, and four independent simulations with different initial configurations and velocities were carried out at 226, 230, and $235 \mathrm{~K}$, respectively, as indicated by the bold multipliers in Table I. At $250 \mathrm{~K}$, constant NPT simulations were carried out at 14 different pressures (from 1 to 12000 bars) for $200 \mathrm{~ns}$ each.

In order to test the validity of our hierarchical two-state scenario, all the calculations were repeated on another widely used water model-the ST2 model. ${ }^{54}$ We simulated 216 ST2 water molecules in a cubic box with periodic boundary conditions by using the hybrid Monte Carlo code developed in Debenedetti's group (http://pablonet.princeton.edu/pgd/html/links.html). Intermolecular interactions were truncated at $7.8 \AA$, and the long-range electrostatic interactions were calculated by using the reaction field method. Constant NPT simulations were performed in a wide range of temperatures and pressures. Equilibration was confirmed in the same way as for TIP5P water. 15 molecular dynamics steps were performed for each Monte Carlo move. Trajectories of $180 \times 10^{6}$ Monte Carlo moves in total were sampled for statistical analyses.

\section{RESULTS}

\section{A. Water's local structure and its impact on local mobility}

\section{Definition of a structural descriptor $\zeta$}

Focusing on water's second shell of nearest neighbors, ${ }^{55,56}$ we introduce the $\zeta$ parameter to measure local translational order. ${ }^{46}$ It is defined as the difference between the distance $d_{j^{\prime} i}$ of the closest neighbor molecule $j^{\prime}$ not H-bonded to molecule $i$ and the distance $d_{j^{\prime \prime} i}$ of the furthest neighbor molecule $j^{\prime \prime} \mathrm{H}$-bonded to molecule $i: \zeta(i)=d_{j^{\prime} i}-d_{j^{\prime \prime} i}$. Two water molecules are regarded as $\mathrm{H}$-bonded only if the $\mathrm{O}-\mathrm{O}$ distance is within $0.35 \mathrm{~nm}$ and the $\mathrm{H}-\mathrm{O} \cdots \mathrm{O}$ angle is less than $30^{\circ} .{ }^{57,58}$ Here we emphasize that considering $\mathrm{H}$-bonding in the characterization of a local structure is a crucial feature of our method. ${ }^{47}$

\section{Two-state feature of $\zeta$ distribution}

As shown in Refs. 29 and 46-48, $\zeta$ has a bimodal distribution, which allows the unambiguous identification of two local structures even under thermal noise.

First we show the local structural characteristics of TIP5P water detected by the parameter $\zeta$ at 1 bar. Figure 1(a) displays two typical local structures: $\rho$ and $S$ states, which are distinguished by $\zeta$. As shown in Fig. 1(b), $\zeta$ has a distinct bimodal feature. The left peak corresponds to a disordered second-shell, with a distribution of $\zeta$ values roughly centered around $\zeta=0$, and considerable shell inter-penetration; we call this the $\rho$ state. The right peak has instead a finite and positive value of $\zeta$, denoting ordered structures; we call this the $S$ state. Figure 1(c) plots the numbers of first-shell $(r<0.35 \mathrm{~nm})$ neighbors and $\mathrm{H}$-bonded neighbors per water molecule as a function of $\zeta$. It can be seen that $S$ state water typically forms H-bonds with all its 4 neighbors, whereas $\rho$ state water only has $\sim 3 \mathrm{H}$-bonds with its 5-6 neighbors [Fig. 1(a)], indicating that the $S$ state is sparser and energetically more stable than the $\rho$ state. Another popular parameter $q$, describing the tetrahedral order, ${ }^{59}$ is plotted as a function of $\zeta$ in Fig. 1(d). The tetrahedral parameter is defined by ${ }^{59,60}$

TABLE I. Total length of molecular dynamics simulations $t$ and reorientational time $\tau_{2}$ for TIP5P water.

\begin{tabular}{lccccccccccccccc}
\hline \hline$T(\mathrm{~K})$ & 226 & 230 & 235 & 240 & 245 & 250 & 260 & 270 & 280 & 300 & 320 & 340 & 360 & 400 & 430 \\
\hline$t(\mathrm{~ns})$ & $\mathbf{3} \times 11000$ & $\mathbf{1 0} \times 3000$ & $\mathbf{4} \times 2430$ & 2000 & 300 & 200 & 50 & 10 & 10 & 2 & 2 & 2 & 2 & 1.5 & 1.2 \\
$\tau_{2}(\mathrm{~ns})$ & 2343 & 413 & 75 & 6.7 & 1.2 & 0.25 & 0.035 & 0.01 & 0.006 & 0.002 & 0.001 & 0.0007 & 0.0005 & 0.0003 & 0.0002 \\
\hline \hline
\end{tabular}




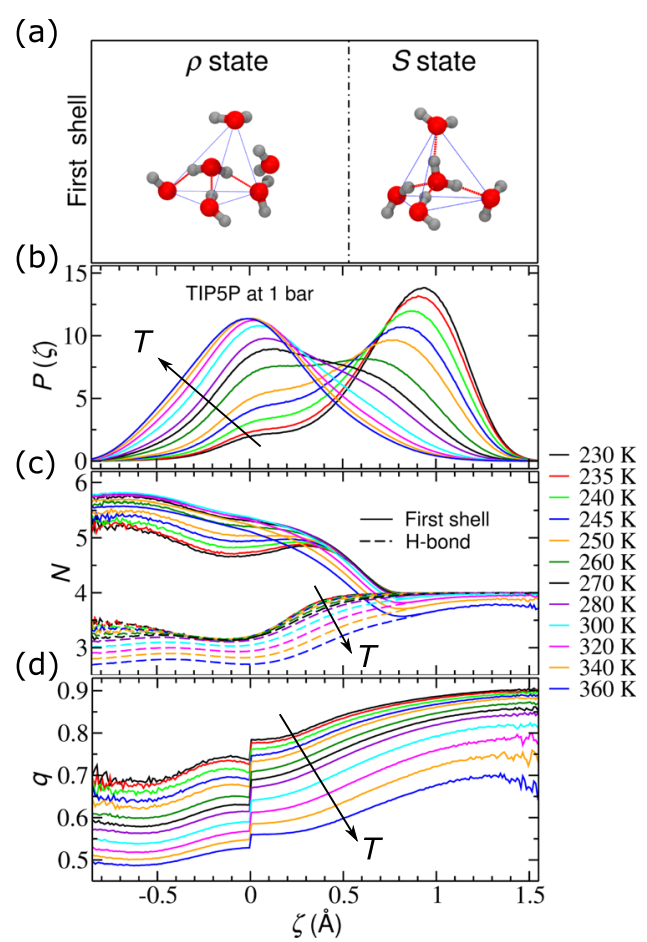

FIG. 1. Local structures of TIP5P water at 1 bar for different temperatures. (a) Typical snapshots of water's local structures for $\rho$ and $S$ states. The dotted red lines represent $\mathrm{H}$-bonds, and the solid blue lines show the tetrahedral structure. (b) Distribution of structural descriptor $\zeta, P(\zeta)$, that characterizes the translational order of water's second shell. $\zeta$ displays a clear bimodal feature and thereby defines two local structures in water: the $\rho$ state (left peak) with a collapsed second shell and the $S$ state (right peak) with a welldefined second shell. (c) Numbers of first-shell ( $r<0.35 \mathrm{~nm}$, solid lines) and H-bonded (dashed lines) neighbors per water molecule as a function of $\zeta$. (d) Tetrahedral parameter $q$ as a function $\zeta$. Overall, as illustrated in (a), the $S$ state has higher order, more H-bonds, and smaller local density than the $\rho$ state. The arrows denote the direction of increasing temperature. Panels in this figure, except for the H-bond number in panel (c), are reproduced with permission from Shi et al., Proc. Natl. Acad. Sci. U. S. A. 115, 9444-9449 (2018) and R. Shi and H. Tanaka, Proc. Natl. Acad. Sci. U. S. A. 115, 1980-1985 (2018). Copyright 2018 Proceedings of the National Academy of Sciences, USA.

$$
q=1-\frac{3}{8} \sum_{i=1}^{3} \sum_{j=i+1}^{4}\left(\cos \psi_{i j}+\frac{1}{3}\right)^{2},
$$

where $\psi_{i j}$ is the angle formed by two vectors pointing from the center molecule to two of its nearest neighbors. The summation runs over all the combinations of the four nearest neighbors. The $S$ state shows higher tetrahedral order than the $\rho$ state because in the $\rho$ state, water penetrates into the first shell, distorting the tetrahedral geometry. The discontinuity at $\zeta=0$ comes from the fact that $q$ is defined by the geometry of nearest rather than $\mathrm{H}$-bonded neighbors so that at $\zeta<0$, the penetrated water will replace the $\mathrm{H}$-bonded one in the calculation of $q$.

Local structures of TIP5P water at $250 \mathrm{~K}$ for different pressures were also analyzed, and the result is shown in Fig. 2. It can be seen that the distribution of structural descriptor $\zeta$ retains its bimodal feature even at high pressure [see Fig. 2(a)]. High pressure pushes water molecules from the second shell into the first shell, and as a result, the fraction of the $S$ state decreases as the pressure is increased. This effect can also be seen in Fig. 2(b) which shows the numbers of first-shell (a)

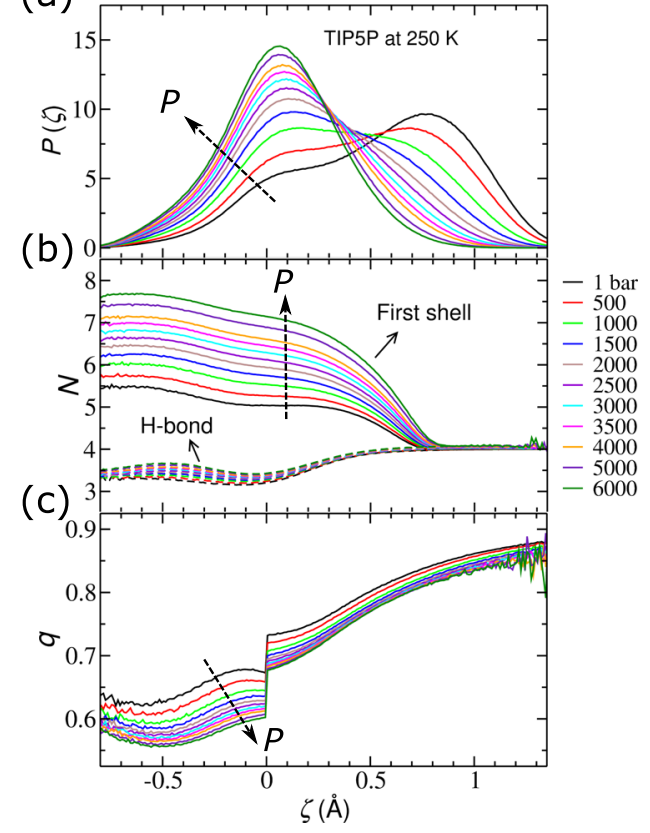

FIG. 2. Local structures of TIP5P water at $250 \mathrm{~K}$ for different pressures. (a) Distribution of structural descriptor $\zeta$. (b) Numbers of first-shell $(r<0.35$ $\mathrm{nm}$, solid lines) and H-bonded (dashed lines) neighbors per water molecule as a function of $\zeta$. (c) Tetrahedral parameter $q$ as a function of $\zeta$. The dashed arrows denote the direction of increasing pressure.

$(r<0.35 \mathrm{~nm})$ and H-bonded water. In contrast to the $S$ state water (high $\zeta$ ) which forms $4 \mathrm{H}$-bonds with 4 neighbors in the first shell, up to 8 water molecules are found in the first shell of the $\rho$ state at high pressures. Figure 2(c) displays the tetrahedral parameter as a function of $\zeta$. In Fig. 2(c), we can see that $S$ state water has a higher tetrahedral order than the $\rho$ state, and for both states, tetrahedral order decreases with pressure.

In a wide temperature and pressure range $\zeta$ distributions show a clear bimodal feature. Because of thermal fluctuation, it is natural to assume that $\zeta$ follows a Gaussian distribution for each state. In fact, we found that at most temperatures and pressures studied in this paper, the $\zeta$ distribution can be fitted by the sum of two Gaussian functions very well. The results at some temperatures and pressures are plotted in Fig. 3. The fraction of the $S$ state was then obtained from the areas of the two Gaussian functions. However, at very low and high temperatures, where one structure predominates in the system, the peaks become slightly asymmetric, vitiating the two-Gaussian fitting. This is because the definition of $\zeta$ puts a strong boundary at high $\zeta$ regions. The boundary comes from the fact that the nearest non-H-bonded water cannot exceed the second nearest non-H-bonded one, or otherwise, it would be replaced by the latter in the calculation of $\zeta$, which makes the right side of the $S$ peak look narrower than its left half [see Fig. 3(a), for example]. To resolve this problem, at very low or high temperatures, we fitted the more symmetric $\rho$ peak by one Gaussian function and the rest was all assigned to the $S$ state. Figure 3(a) shows the two-state decomposition by one Gaussian fit to data at $\zeta<0.15 \AA$ at 1 bar and $230 \mathrm{~K}$, and Figs. 3(b)-3(d) display the two-state decomposition by two Gaussians at different pressures and temperatures. Then the fraction of the $S$ 

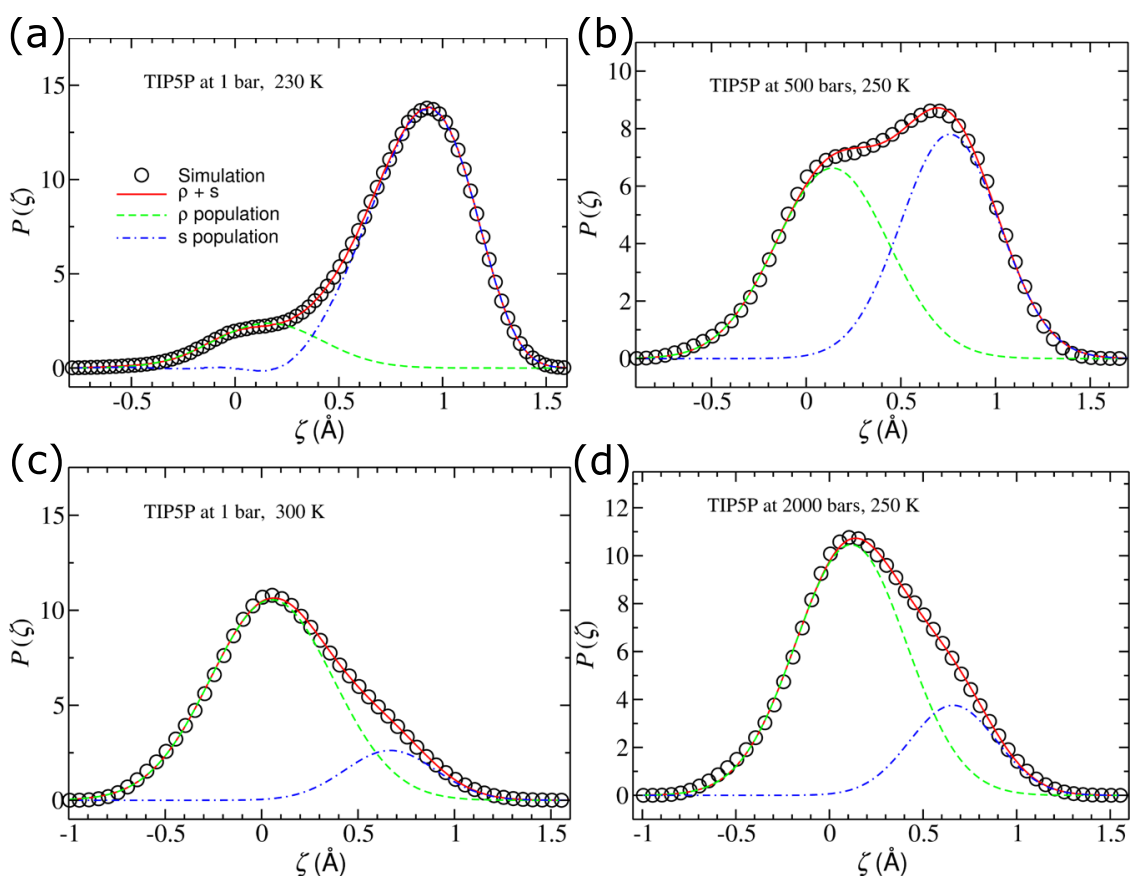

FIG. 3. Decomposition of the distribution of structural descriptor $\zeta$ into two states. (a) $230 \mathrm{~K}$ and $1 \mathrm{bar}$, (b) $250 \mathrm{~K}$ and 500 bars, (c) $300 \mathrm{~K}$ and 1 bar, and (d) $250 \mathrm{~K}$ and 2000 bars. Circles denote simulation data. Red solid lines are the fitting results. Green broken lines and blue dotted-dashed lines represent the $\rho$ and $S$ state distributions, respectively. state is defined as the static order parameter $s$ in the two-state model.

Two distinct types of local structures can thus be identified microscopically - a more ordered, sparser $S$ state and a less ordered, denser $\rho$ state, with their populations varying with temperature and pressure. Considering the large structural fluctuations, we should use "two types of structures" rather than "two structures," but hereafter, we use the latter for simplicity. We note that the existence of two such local structures in water is supported by many experiments, such as Raman spectroscopy, ${ }^{61-63}$ femtosecond mid-IR pumpprobe spectroscopy, ${ }^{64}$ time-resolved optical Kerr effect spectroscopy, ${ }^{65} \mathrm{x}$-ray absorption, ${ }^{66}$ and emission spectroscopies ${ }^{67}$ (see recent reviews by Nilsson and Pettersson ${ }^{4}$ and by Gallo et $a l .^{5}$ ), in which signatures of two structures were detected, although the details of the two structures are more difficult to be identified experimentally.

\section{Two-state feature in real space}

Figure 4 displays two typical local structures detected by $\zeta: \rho$ and $S$ states [Fig. 4(a)]. We showed ${ }^{46}$ that $\zeta$ gives us a quantitative description of local structural ordering in water at the molecular level and can thus describe the thermodynamic anomalies. For example, we plot in Figs. 4(b)-4(f) different fields for a sample configuration for the TIP5P model at 1 bar and $250 \mathrm{~K}$, where every molecule is colored in red if the field is low or blue if the field is high. A high degree of correlation (correlation factor $\approx 0.80$ ) is evident between the $\zeta$ field [Fig. 4(b)] and the inverse of local density field [Fig. 4(c)]. While thermodynamic properties show a high degree of correlation with $\zeta$, at first sight, the spatial distribution of dynamical quantities is instead weakly correlated with the $\zeta$ field. This is shown, for example, in Figs. 4(e) and 4(f), where the particles are colored according to their inverse translational and rotational mobility, respectively.
In our previous phenomenological two-state model, we used the same order parameter to describe thermodynamic and viscosity anomalies. ${ }^{32-34}$ But more recent studies on the correlation between dynamics and structural order have shown that the dynamics cannot be determined locally. ${ }^{68}$ The microscopic dynamics of a water molecule in a mixture of the two states must be strongly affected by the composition of its first-shell neighbors under the constraint of H-bonding, so the local environment of each molecule has to be taken into account. This hierarchy is incorporated in our hierarchical two-state model. ${ }^{29}$ More specifically, we coarse-grain the $\zeta$ field up to the first shell of nearest neighbors. The spatial distribution of the coarse-grained field $\zeta^{C G}$ is shown in Fig. 4(d), which this time shows a high degree of correlation (correlation factor $\approx 0.53$ ) with both the inverse translational [Fig. 4(e)] and rotational [Fig. 4(f)] mobility field. It is clear that the $\zeta$ field without coarse-graining [Fig. 4(b)] does not have a good correlation with neither the translational nor rotational mobility field [Figs. 4(e) and 4(f)], indicating the fundamental deficiency of all previous two-state approaches for dynamics. ${ }^{32-34,40,42}$ It is remarkable that the $\zeta$ field and the coarse-grained $\zeta^{\mathrm{CG}}$ field have a direct correlation with local volume and mobility, respectively, at the molecular level. This strongly indicates that the dynamics of a water molecule is controlled by its local environment up to the first-shell neighbors. In the following, we will make this correspondence more precise, by showing that a hierarchical two-state model of $\zeta^{C G}$ is able to quantitatively predict water's dynamical anomalies.

\section{B. Two-state description of dynamic anomalies}

\section{Theoretical framework}

Our hierarchical two-state model of dynamic anomalies is obtained by dividing the molecules into two states in terms 
(a)

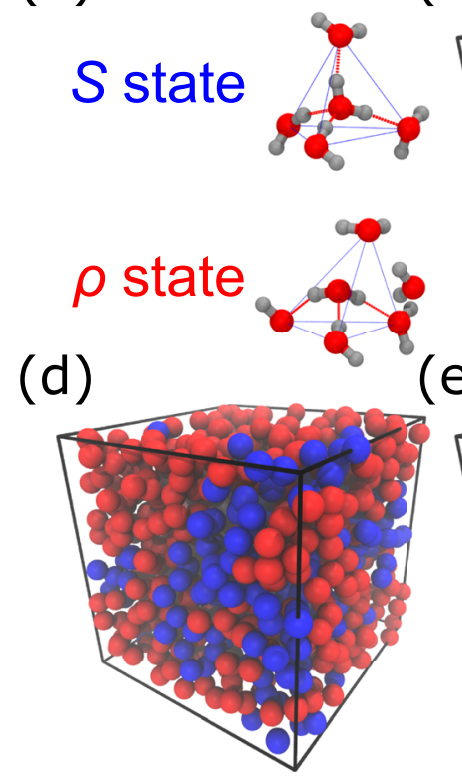

(b)

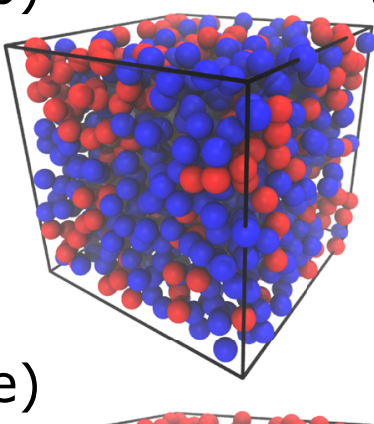

(c)
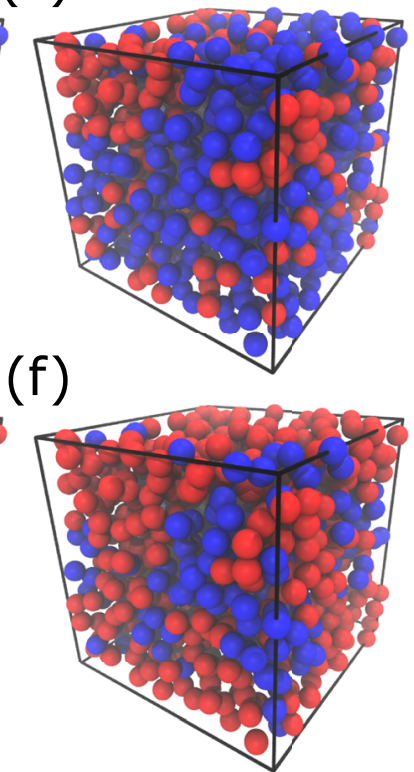

FIG. 4. Water's local structures and their correlations to local density and mobility. (a) Typical snapshots of water's local structures for $\rho$ and $S$ states. [(b)-(f)] Different fields for a sample configuration for the TIP5P water at 1 bar and $250 \mathrm{~K}$. All molecules are colored in red if the field is low or blue if it is high. (b) $\zeta$ field. (c) Inverse local density field (6- $N_{\mathrm{fs}}$, where $N_{\mathrm{fs}}$ is the number of first-shell neighbors). (d) $\zeta$ CG field. (e) Inverse translational mobility field (1$\left.\Delta r_{\max }\left(\tau_{4}\right)\right)$, where $\Delta r_{\max }\left(\tau_{4}\right)$ is the maximum distance one molecule travels during a time period of $\tau_{4}$. (f) Inverse rotational mobility field (1- $\left.\Delta \phi_{\text {max }}\left(\tau_{4}\right)\right)$, where $\Delta \phi_{\max }\left(\tau_{4}\right)$ is the maximum angle one molecule rotates during a time period of $\tau_{4}\left(\tau_{4}\right.$ : dynamic heterogeneity time scale. See also Appendix A). In (b)-(d), a simple low-pass filter by performing running time average of each field over $\tau_{4}$ is utilized to remove thermal noise. In [(b)-(c)] and [(d)-(f)], $s=57 \%$ and $s^{\mathrm{D}}=26 \%$ molecules with higher field are colored in blue, respectively. This difference between the static and dynamic order parameters is the consequence of the hierarchical nature of the impact of local structural ordering on static and dynamic quantities. Panel (a) is reproduced with permission from Fig. 1 of Shi et al., Proc. Natl. Acad. Sci. U. S. A. 115, 9444-9449 (2018). Copyright 2018 Proceedings of the National Academy of Sciences, USA. The data shown in other panels were calculated by using a randomly selected configuration which is different from the one used in Fig. 1 of Ref. 29.

of the activation energy by using $\zeta$ coarse-grained up to the first shell. We refer to these states with low $\left(E_{\mathrm{a}}^{\rho}\right)$ and high activation energy $\left(E_{\mathrm{a}}^{S}\right)$ as dynamic $\rho$ and $S$ states, respectively. The fraction of the dynamic $S$ state is denoted by $s^{\mathrm{D}}$. A dynamic quantity $X$ is then expressed by the generalized Arrhenius law as

$$
X=X_{0} \exp \left[\frac{E_{\mathrm{a}}^{\rho, X}+\Delta E_{\mathrm{a}}^{X} \cdot s^{\mathrm{D}}}{k_{\mathrm{B}} T}\right],
$$

where $\Delta E_{\mathrm{a}}^{X}=E_{\mathrm{a}}^{S, X}-E_{\mathrm{a}}^{\rho, X}$ is the activation energy difference between dynamic $S$ and $\rho$ states, $X_{0}$ is the prefactor, and $k_{\mathrm{B}}$ is the Boltzmann constant. This expression has a form similar to that in Refs. 32 and 34. As discussed above, however, we use $s^{\mathrm{D}}$ instead of $s$ in Eq. (2) unlike in Refs. 32 and 34, which highlights the importance of coarse-graining.

A hidden assumption behind Eq. (2) is that the lifetime of dynamic $\rho$ and $S$ states should be much shorter than the characteristic dynamical time scale of water so that we can treat the average of the activation energies as the effective activation energy. ${ }^{34}$ Although this assumption is difficult to verify experimentally, in simulations we can directly confirm it by the fact that the lifetime of dynamic states is approximately two orders of magnitude shorter than water's reorientational time. Below we show evidence supporting this assumption.

\section{Local structure relaxation}

Here we characterize the dynamics of water's local structure spanning over a wide time range. By using the structural descriptor $\zeta$, we can define local structures for instantaneous configurations. These structures evolve and transform between each other with time. The dynamics of water's local structures might not be important for static properties, but essential for water's dynamics. In the two-state scenario, dynamic properties of each pure state follow the classical Arrhenius law. The apparent non-Arrhenius behavior comes from an effective activation energy that varies with temperature and pressure [see Eq. (2)]. If the lifetime of local structures is much shorter than the dynamic time scale, then it is natural to write the effective activation energy as a sum of two-state components. Having a more ordered structure, the dynamic $S$ state has a larger activation energy than the $\rho$ state. Therefore the slowing down of dynamics can be naturally interpreted by the Arrhenius law with an effective activation energy increasing with the increase of the concentration $s^{\mathrm{D}}$ of the dynamic $S$ state upon cooling. An assumption behind this idea is that the lifetime of two states should be much shorter than the dynamic time scale. ${ }^{32-34}$ The time correlation function was used to characterize the time scales in water. The kinetics of two states can be estimated from the time correlation functions of coarse-grained structural descriptor $\zeta^{\mathrm{CG}}$ by

$$
C_{\zeta^{\mathrm{CG}}}(t)=\frac{\left\langle\zeta^{\mathrm{CG}}(t) \zeta^{\mathrm{CG}}(0)\right\rangle-\left\langle\zeta^{\mathrm{CG}}\right\rangle^{2}}{\left\langle\left(\zeta^{\mathrm{CG}}\right)^{2}\right\rangle-\left\langle\zeta^{\mathrm{CG}}\right\rangle^{2}} .
$$

Figure 5 displays the time correlation functions of structural descriptor $\zeta^{\mathrm{CG}}$, which show clear a two-step decay at various temperatures. Similar to the self-intermediate 


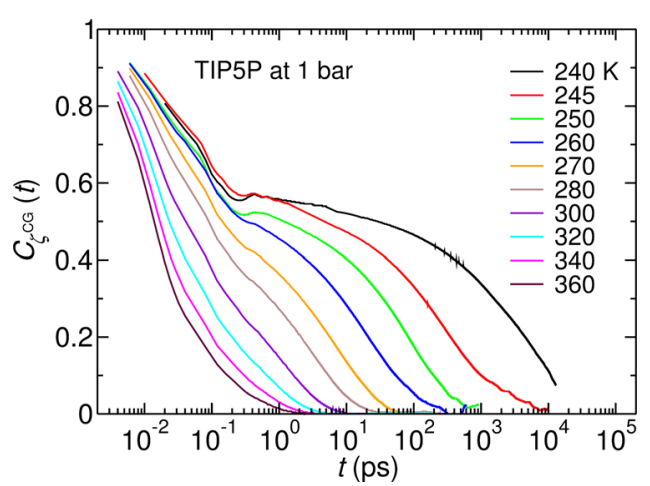

FIG. 5. Time correlation function of coarse-grained structural descriptor $\zeta^{\mathrm{CG}}$ at 1 bar.

scattering function, the two-step decay indicates two modes of local structure relaxation in water, with different time scales. In order to see the relaxation directly, in Fig. 6(a), we plot the time evolution of local structure index $I(t)$ ( 1 for the dynamic $S$ state and 0 for the dynamic $\rho$ state) of five randomly chosen molecules from our simulation at 1 bar and $250 \mathrm{~K}$. The observation was performed for $0.5 \tau_{2}$. Clearly, during half the rotational time scale $\tau_{2}$, the local structure fluctuates quite fast. This fast mode may be decomposed into two components:
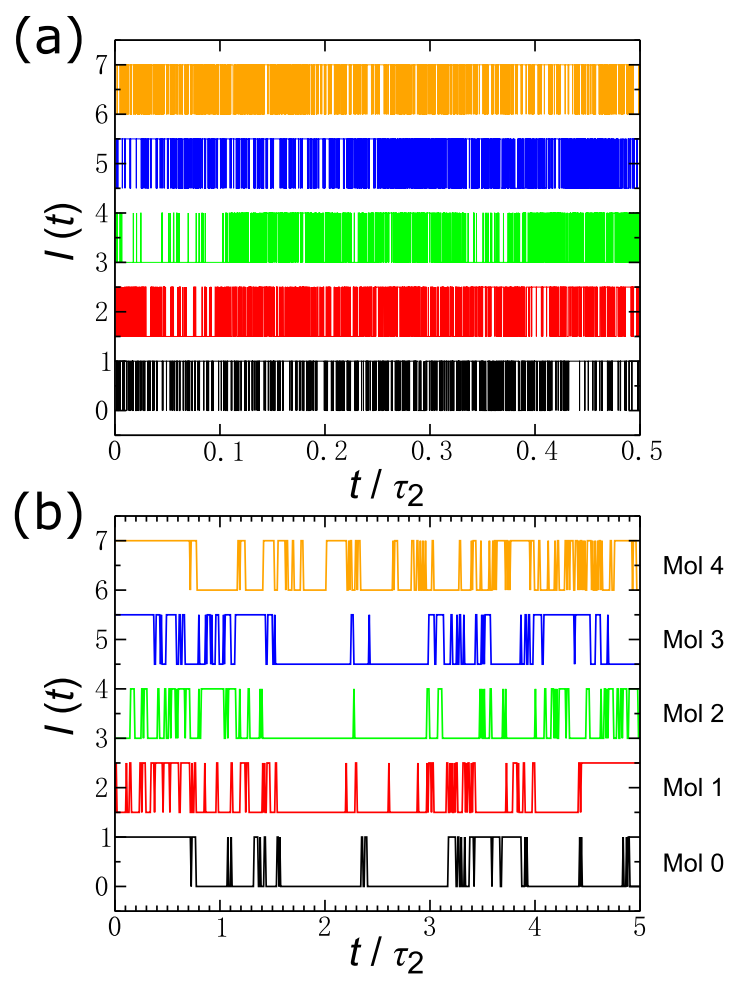

FIG. 6. Evolution of local structures of five randomly chosen water molecules at 1 bar and $250 \mathrm{~K}$. (a) Instantaneous local structures with a time resolution of $20 \mathrm{fs}$. Local structure index $I(t)$ takes 0 or 1 , representing dynamic $\rho$ and $S$ states, respectively. The values of $I(t)$ were shifted upward to avoid overlap. A threshold $\zeta_{c}^{\mathrm{CG}}=0.7 \AA$ was applied for determining dynamic $\rho$ and $S$ states for each molecule. If $\zeta^{\mathrm{CG}}>\zeta_{c}^{\mathrm{CG}}$, then it is assigned as the dynamic $S$ state and vice versa. (b) "Averaged local structures" with a time resolution of $2 \mathrm{ps}$. A simple low-pass filter by performing rolling average of $I(t)$ over 2 ps was employed to obtain an "averaged local structure" index. The reorientational time $\tau_{2}=246.5 \mathrm{ps}$ was determined by a stretched exponential fitting to the reorientational time correlation function $C_{2}(t)$ at 1 bar and $250 \mathrm{~K}$. one comes from thermal fluctuations that can lead to switching between the two states separated by the threshold values of the structural descriptors $\left(\zeta\right.$ and $\zeta^{\mathrm{CG}}$ ) even without any intrinsic change in the local H-bond network around a central molecule and the other comes from high-frequency librational motion, which leads to rearrangement of the H-bond network at a short (subpicosecond) time scale. The former should not be regarded as true switching between the two states, but the latter should reflect intrinsic structural transformation. To illustrate the latter process, we plot in Fig. 7 the temporal change in the local structure around a randomly chosen water molecule during $2.5 \mathrm{ps}\left(\cong 0.01 \tau_{2}\right)$. It clearly shows that for both structural descriptors $\left(\zeta\right.$ and $\zeta^{\mathrm{CG}}$ ), the number of $\mathrm{H}$-bond neighbors and the number of first-shell neighbors indeed change during this time period. The snapshots in the inset show a typical example of how librational motion leads to the temporal change in the H-bond network structure around the center molecule. All these results clearly indicate that water's local structure intrinsically fluctuates between the two states at a subpicosecond time scale, which confirms our assumption that two states' lifetime, defined by the fast mode, is much shorter than the dynamic time scale $\tau_{2}$. This fast fluctuation is detected as the fast decay mode of the time correlation function of $\zeta^{\mathrm{CG}}$ we observed in Fig. 5. If we filter out the fast fluctuation by a low-pass filter, there remains the low-frequency structure fluctuation, as shown in Fig. 6(b). This indicates the presence of long-lived local structures in the dynamic time scale $\tau_{2}$, corresponding to the slow decay, as we observed in Fig. 5. This low-pass filtering is effectively the same operation as a time analysis of the $\mathrm{H}$-bond network employed to find stable H-bonds. ${ }^{69}$ We note that a similar two-step decay was also observed for non-coarse-graining structural descriptor $\zeta$.

\section{Key time scales in water}

Here we systematically consider all the relevant time scales for both structural and dynamical properties. The structural time scale can be extracted from the time correlation function of the $\zeta^{\mathrm{CG}}$ field, introduced in Eq. (3). Decaying by two steps, the time correlation function $C_{\zeta^{\mathrm{CG}}}(t)$ defines two time scales of local structure relaxation modes, which can be estimated from a two-stretched-exponential fitting,

$$
C_{\zeta^{\mathrm{CG}}}(t)=k \exp \left[-\left(\frac{t}{\tau_{\zeta^{\mathrm{CG}}}^{\mathrm{f}}}\right)^{\beta^{\mathrm{f}}}\right]+(1-k) \exp \left[-\left(\frac{t}{\tau_{\zeta^{\mathrm{CG}}}^{\mathrm{s}}}\right)^{\beta^{\mathrm{s}}}\right],
$$

where $\tau_{\zeta^{\mathrm{CG}}}^{\mathrm{f}}$ and $\tau_{\zeta^{\mathrm{CG}}}^{\mathrm{s}}$ are the time scales of fast and slow modes, respectively.

As for dynamical time scales, the rotational motion is usually described by a second Legendre polynomial of the dipole-dipole time correlation function

$$
C_{2}(t)=\frac{1}{2}\left[3\langle\boldsymbol{\mu}(t) \boldsymbol{\mu}(0)\rangle^{2}-1\right],
$$

where $\boldsymbol{\mu}(t)$ is the molecular dipole moment at time $t . C_{2}(t)$ typically shows non-exponential decay, and the reorientational time scale $\tau_{2}$ can be calculated from a stretched-exponential fitting of $C_{2}(t)$, 


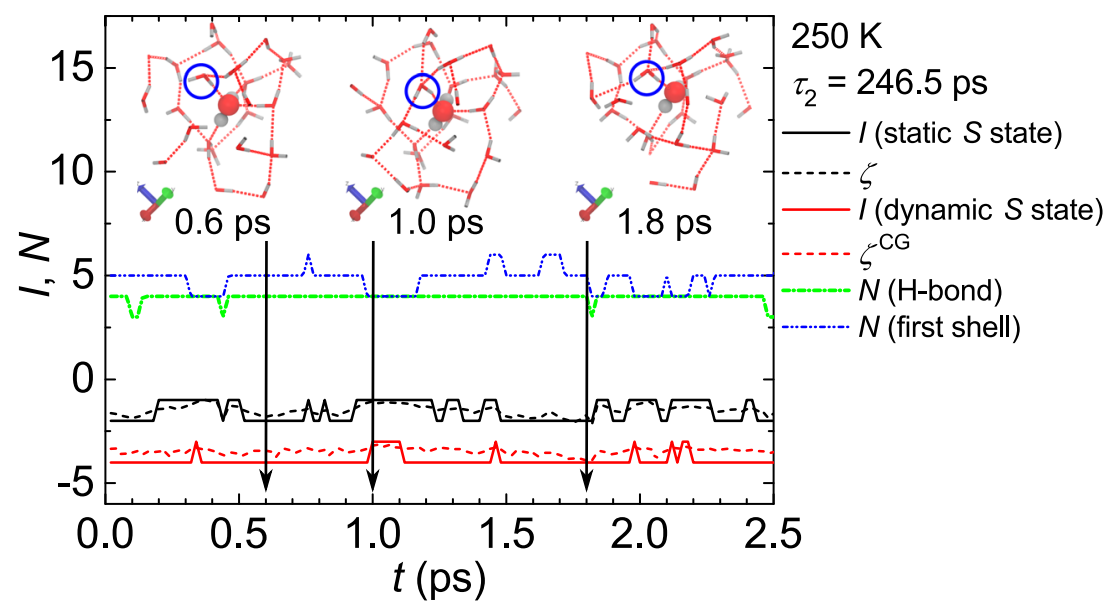

FIG. 7. Short-time local structure fluctuation around a randomly chosen water molecule during $2.5 \mathrm{ps}\left(\cong 0.01 \tau_{2}\right)$ at 1 bar and $250 \mathrm{~K}$ (see also the Supplementary Movie). The local structure index I for the static $S$ state and $\zeta$ (in $\AA$ ) were shifted downward by -2 , and the local structure index I of the dynamic $S$ state and $\zeta$ CG were shifted downward by -4 for clarity. A threshold $\zeta=0.56 \AA$ and $\zeta_{c}^{\mathrm{CG}}=0.7 \AA$ was applied for determining static and dynamic $S$ states, respectively. We note that these choices of the thresholds allow us to detect locally favored structures individually, whose populations agree well with $s$ obtained by decomposition of $\zeta$ distribution (Fig. 3) and $s^{\mathrm{D}}$ estimated by counting the number of ordered neighbors (Fig. 10). Insets are snapshots of the selected molecule (shown by balls) and its surrounding neighbors (shown by sticks) up to the second shell (within $5.5 \AA$ from the central one) at $0.6,1.0$, and 1.8 ps with H-bonds shown by red dotted lines. The blue circles highlight one molecule whose librational motion at subpicosecond time leads to the change in the H-bond network structure near the selected molecule.

$$
C_{2}(t)=a \cdot \exp \left[-\left(\frac{t}{\tau_{2}}\right)^{\beta}\right],
$$

where $a$ is a constant and $\beta$ is the stretching parameter. Dynamic heterogeneity time scale $\tau_{4}$ defines another important time scale in water, where dynamic heterogeneity (characterized by $\chi_{4}$ ) maximizes (see Appendix A). In Fig. 8, we plot $\tau_{4}$ with $\tau_{2}$ as a function of temperature and find that they have almost the same time scale.

In Fig. 8, we compare all the extracted structural and dynamic time scales as a function of temperature. At very low temperatures, short structure relaxation times are not shown because short-time motions were not recorded at low temperatures. From the plot, we can see that the slow mode relaxation time $\tau_{\zeta^{\mathrm{CG}}}^{\mathrm{s}}$ is roughly in the same time scale of $\tau_{2}$ and $\tau_{4}$. This is consistent with the jump mechanism of water reorientation, by which the rotation of water is coupled to the penetration

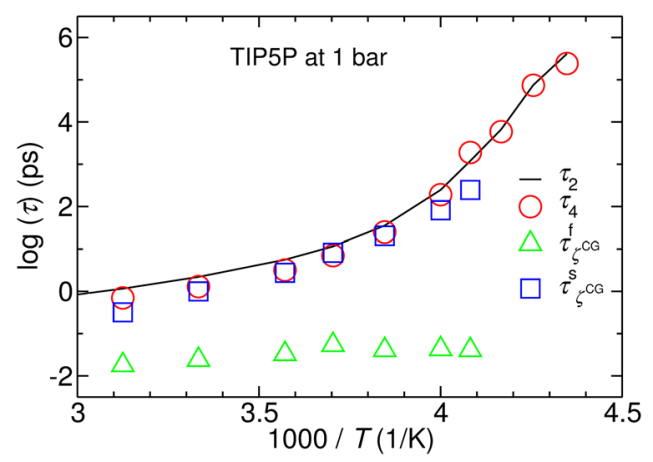

FIG. 8. Structural and dynamic time scales in TIP5P water at 1 bar. Reorientational time scale $\tau_{2}$ was determined by a stretched exponential fitting to the reorientational time correlation function $C_{2}(t) . \tau_{4}$ characterizes a time scale of dynamic heterogeneity, where the four-point susceptibility maximizes. The local structure relaxation is characterized by two time scales: $\tau_{\zeta^{\mathrm{CG}}}^{\mathrm{f}}$ and $\tau_{\zeta^{\mathrm{CG}}}^{\mathrm{s}}$ for fast and slow relaxations, respectively. of its first shell: a water molecule rotates through breaking old $\mathrm{H}$-bonds and forming new $\mathrm{H}$-bonds with penetrated water molecules. ${ }^{70}$ On the other hand, the fast mode $\left(\tau_{\zeta \mathrm{CG}}^{\mathrm{f}}\right)$ shows a much shorter time scale than the dynamic time scale by about two orders of magnitude. Moreover, $\tau_{\zeta \mathrm{CG}}^{\mathrm{f}}$ is comparable to the molecular libration time scale $(\sim 0.01 \mathrm{ps})$, which, along with its weak temperature dependence, indicates a link of the fast structure relaxation to intermolecular vibration and libration.

The time scales in water can thus be summarized as $\tau_{\zeta^{\mathrm{CG}}}^{\mathrm{f}} \ll \tau_{\zeta^{\mathrm{CG}}}^{\mathrm{s}} \sim \tau_{4} \sim \tau_{2}$. These time scale relations underpin the applicability of the two-state model to the dynamics of water: On the one hand, the fast structure relaxation time $\tau_{\zeta \mathrm{CG}}^{\mathrm{f}}$ allows an Arrhenius description of water's dynamic properties with an effective activation energy [see Eq. (2)]. On the other hand, as a prerequisite of dynamic heterogeneity, the slow structure relaxation time $\tau_{\zeta^{\mathrm{CG}}}^{\mathrm{s}}$ allows a link between local structure and dynamics. The latter is essential for understanding the dynamic heterogeneity in water, which will be discussed later.

\section{Dynamic S state}

As mentioned above, for the study of the dynamics, it is crucial to include the properties of the local environment (firstshell neighbors) in the description of the order parameter. In other words, the definition of a dynamic state should involve the local environment in which the water molecule is embedded. It is thus natural to define dynamic states by using a coarsegrained structural descriptor $\zeta^{\mathrm{CG}}$ that includes the structure information up to the third shell. In Fig. 9, we plot the coarsegraining numbers of first-shell and $\mathrm{H}$-bonded water as a function of $\zeta^{\mathrm{CG}}-\zeta_{\mathrm{c}}^{\mathrm{CG}}$, where $\zeta_{\mathrm{c}}^{\mathrm{CG}}$ is a threshold above which water is defined as the dynamic $S$ state. We note that all the coarsegraining was performed by taking an average over the first 


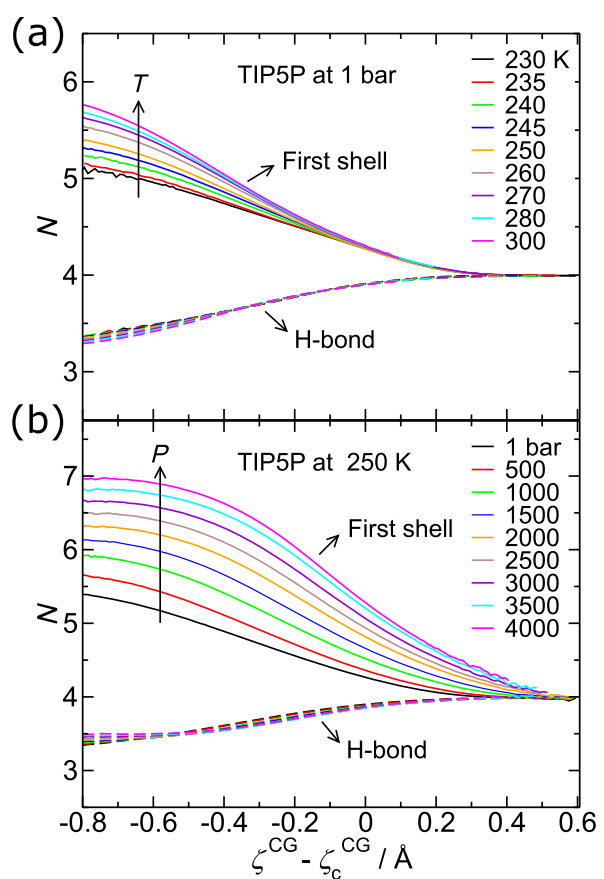

FIG. 9. Numbers of first-shell and H-bonded water as a function of $\zeta^{\mathrm{CG}}$. A water molecule is defined as the dynamic $S$ state if $\zeta^{\mathrm{CG}}>\zeta_{\mathrm{c}}^{\mathrm{CG}}$ : (a) 1 bar and (b) $250 \mathrm{~K}$. In (a), the curves for high temperatures were slightly shifted upwards to reach a reasonable value of four at a high $\zeta^{\mathrm{CG}}$ regime and the threshold $\zeta_{\mathrm{c}}^{\mathrm{CG}}$ is independent on temperature. In (b), the threshold $\zeta_{\mathrm{c}}^{\mathrm{CG}}$ becomes pressure dependent. In this plot, the numbers of first-shell and $\mathrm{H}$-bonded water were coarse-grained in the first shell.

shell. We can see that at a large $\zeta^{\mathrm{CG}}$ regime, the number of firstshell water equals the number of $\mathrm{H}$-bonded water, indicating a well-defined first shell structure. Instead, the number of firstshell water exceeds the number of $\mathrm{H}$-bonded water in the small $\zeta^{\mathrm{CG}}$ regime, suggesting the penetration of a water molecule into the first shell. We find that $\zeta_{\mathrm{c}}^{\mathrm{CG}}$ does not have a sensible temperature dependence. The number of hydrogen bonded molecules [dashed curves in Fig. 9(a)] falls on a master curve when plotted against $\zeta^{\mathrm{CG}}-\zeta_{\mathrm{c}}^{\mathrm{CG}}$ (the only exception is the curves at temperatures above melting, which require a slight vertical shift to fall on the master curve). On the other hand, $\zeta_{\mathrm{c}}^{\mathrm{CG}}$ shows some pressure dependence. This can be determined by requiring that the number of $\mathrm{H}$-bonded molecules is 4 at high values of $\zeta^{\mathrm{CG}}$. Figure 9(b) shows that if this criterion is employed, all hydrogen-bonded curves again fall on a master curve. The master curve starts to decrease just at the threshold $\zeta_{\mathrm{c}}^{\mathrm{CG}}$, implying an important feature of the dynamic $S$ state: it keeps nearly four $\mathrm{H}$-bonds with its neighbors, indicating high tetrahedrality.

Besides coarse-graining, another way to define dynamic states is detecting the structure of not only the molecule but also its neighbors simultaneously. An effective method is simply counting the number of $S$-state neighbors. We confirm that a $S$-state water protected by 3 or more $S$-state neighbors can hardly rotate, but those protected by 2 or less $S$-state neighbors can easily make a rotation. It is therefore natural to define the dynamic $S$ state as the clustered $S$ state $-S$ water with 3 or $4 S$ neighbors $\left(s_{3}+s_{4}\right)$, where we define $s_{i}$ as an $S$ state having $i S$ neighbors. This is rationalized by the following microscopic consideration on the jump mechanism of water reorientation. It is known ${ }^{70,71}$ that the rotation of water is coupled to the penetration of its first shell by water molecules: a water molecule rotates through breaking old H-bonds and forming new H-bonds with penetrated water molecules. Thus, the presence of at least two penetrating water molecules nearby, i.e., the presence of more than two $\rho$ states around the central $S$ state, is necessary for the quick rotation of the central water molecule. In order to compare the two methods, we plot the fractions of the dynamic $S$ state detected by these two methods in Fig. 10. Clearly, the two methods suggest almost the same temperature dependence of $s^{\mathrm{D}}$. Moreover, the dynamic $S$ states detected by these two ways are heavily overlapped in real space, indicating the consistency in the definition of dynamic states by coarse-graining and cluster methods and thus the robustness of the estimation of $s^{\mathrm{D}}$.

In Figs. 13(e) and 14(e), we plot dynamic $s^{\mathrm{D}}$ together with thermodynamic $s$, which is obtained by the decomposition of $P(\zeta)$ (see Fig. 3). Since the dynamic $S$ state comprises only those molecules whose neighbors are predominantly in the static $S$ state, we can see that the amount of the dynamic $S$ state is much less than that of the $S$ state itself, but still shows clear two-state features: it vanishes at high temperatures and gradually approaches one at low temperatures. In fact, we found that in both TIP5P and ST2 water, the fraction of the dynamic $S$ state can also be well described by Eq. (1) (by replacing the parameters $\Delta E, \Delta \sigma$ and $\Delta V$ by $\Delta E^{D}, \Delta \sigma^{D}$ and $\Delta V^{D}$, respectively), as shown by the solid line in Fig. 10.

\section{Dynamic bimodality as a result of two states under the influence of neighbors}

As shown above, the spatial coarse-graining of $\zeta$ up to first-shell neighbors to define the dynamic order parameter $s^{\mathrm{D}}$ is of fundamental importance for a two-state description of water's dynamic anomalies. This comes from the intrinsic difference between static and dynamic quantities: the former

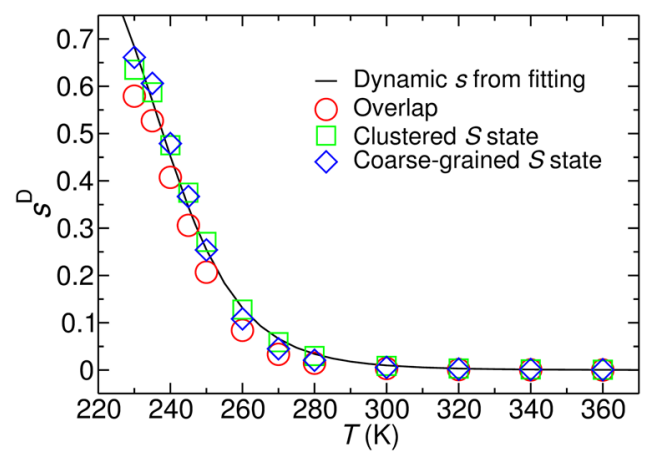

FIG. 10. Fraction of the dynamic $S$ state as a function of temperature at 1 bar for TIP5P water. The dynamics of water molecules depends on the structure of not only itself but also its neighbors. There are two simple ways to define the dynamic $S$ state by considering the local environment of a water molecule. (1) Dynamic $S$ state can be defined as a coarse-grained $S$ state (blue diamonds) by using the threshold $\zeta^{\mathrm{CG}}>\zeta_{\mathrm{c}}^{\mathrm{CG}}$. (2) Dynamic $S$ state can also be defined as a clustered $S$ state (green squares) with itself, and 3 or more neighbors are all static $S$ states. The fraction of water molecules satisfying both definitions is given by red circles in the plot. The solid line is the fraction of the dynamic $S$ state obtained from two-state fitting [Eq. (1)]. It can be seen that both definitions give reasonable and consistent estimation of the dynamic $S$ state. 
can be defined instantaneously, whereas the latter can only be determined as time passes. Due to the fast relaxation mode of locally favored structures (Figs. 5 and 6), they change the structure frequently during a dynamic time scale, e.g., $\tau_{4}$. However, thanks to the slow mode, the locally favored structures survive during the dynamic time scale and are thus able to control dynamics in a statistical way: the higher the probability that a molecule can continue to be in the $S$ state, the larger the effective activation energy, and the slower the motion. In Fig. 11(a), we show the survival probability of the $S$ state, $P_{\mathrm{S}}^{*}$, during $\tau_{4}$ for different initial local environments $\left[\rho\right.$ and $s_{i}(i=0-4)$ states]. Interestingly, we found that these different initial structures are naturally divided by the average probability $\left\langle P_{S}^{*}\right\rangle(T)$ into two groups: clustered (solid blue symbols) and non-clustered (open red symbols) $S$ states.
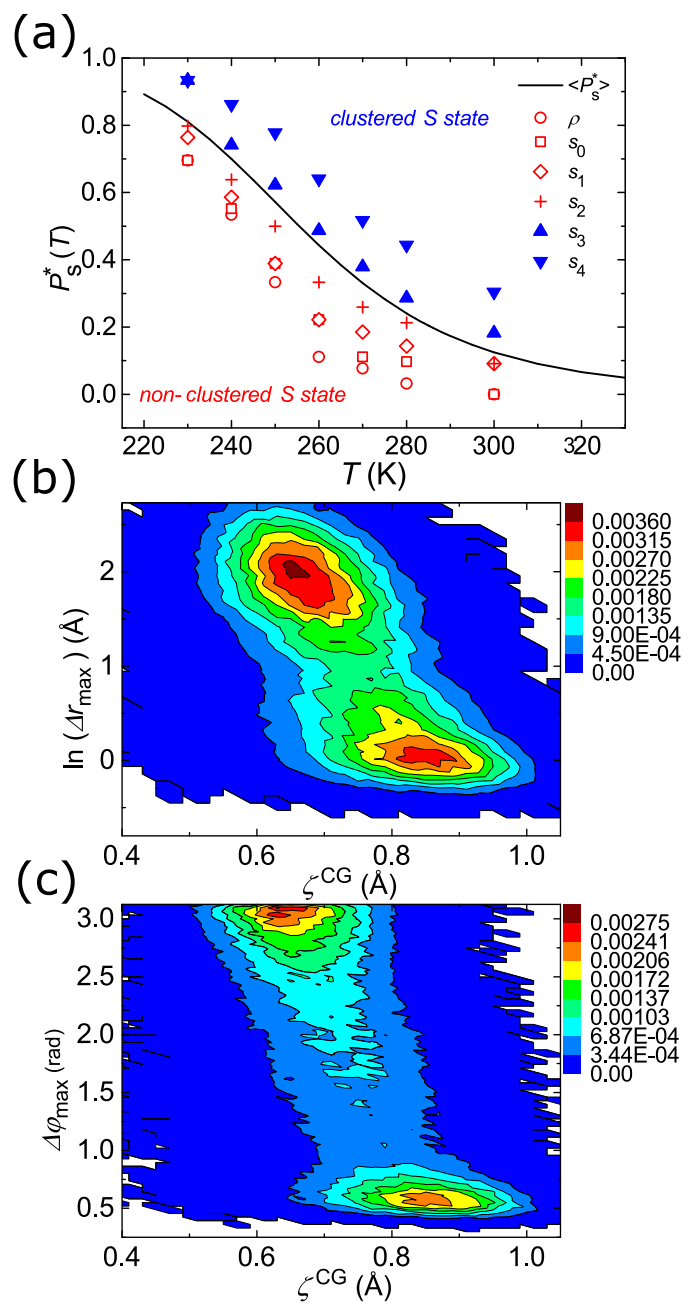

FIG. 11. (a) $S$-state survival probability $P_{S}^{*}$ as a function of temperature at 1 bar for different initial local environments $\left[\rho\right.$ and $\left.s_{i}(i=0-4)\right]$ for TIP5P water. $s_{i}$ denotes the initial environment of a $S$ state with $i S$-state neighbors. The black lines represent the long-time average of $P_{S}^{*},\left\langle P_{S}^{*}\right\rangle=s$, which separates the clustered and non-clustered $S$ state. (b) Correlation map of translational mobility (maximum translation distance $\Delta r_{\max }\left(\tau_{4}\right)$ ) and $\zeta^{\mathrm{CG}}$ during the characteristic time scale of dynamic heterogeneity, $\tau_{4}$, at 1 bar and $235 \mathrm{~K}$ for TIP5P water. (c) Correlation map of rotational mobility (maximum rotation angle $\left.\Delta \phi_{\max }\left(\tau_{4}\right)\right)$ and $\zeta^{\mathrm{CG}}$ during $\tau_{4}$ at 1 bar and $235 \mathrm{~K}$ for TIP5P water. A simple low-pass filter by running time average of $\zeta^{\mathrm{CG}}$ over $\tau_{4}$ is utilized to remove thermal noise. Panel (b) is reproduced with permission from Fig. S2 in Shi et al., Proc. Natl. Acad. Sci. U. S. A. 115, 9444-9449 (2018). Copyright 2018 Proceedings of the National Academy of Sciences, USA.
The clustered $S$ state $\left(s_{3}+s_{4}\right)$ that is protected by 3 or 4 $S$-state neighbors can survive longer than the average, whereas the non-clustered $S$ state without enough protection by $S$ neighbors cannot. As a result, the clustered and non-clustered $S$ states show essentially different dynamics. In Figs. 11(b) and 11(c), we can see that water's dynamics indeed shows a clear bimodal feature and well correlates with the coarse-grained $\zeta$, in agreement with Figs. 4(d)-4(f). This result not only supports the validity of two-state description of water's dynamics but also provides a fundamental understanding of the importance of spatial coarse-graining.

\section{Evidence for the unimportance of cooperativity}

As mentioned in Sec. II, we have found that ignoring cooperativity (i.e., setting $J=0$ ) still allows us to describe both the thermodynamic and dynamic anomalies of water. This is expected as all state points considered in this work are far-away from the critical point.

In this section, we provide additional evidence by directly measuring the degree of short-range order in the mixture. As shown in Fig. 10, the definition of the dynamic $S$ state from $S$ cluster $\left(s_{3}+s_{4}\right)$ is equivalent to that from the coarsegrained structural descriptor $\zeta^{\mathrm{CG}}$. To check whether the distribution of these clusters is random or cooperative, we make the following analysis. In a system of $N$ water molecules, we randomly assign $n=s \cdot N$ water molecules to the $S$ state. From these randomly generated concentration distributions, we then compute the fraction of dynamic $S$ states by counting the molecules in the $S$ state that are surrounded by at least three nearest neighbors that also belong to the $S$ state $\left(s_{3}+s_{4}\right)$. In Fig. 12, we show that the fraction of $s_{3}+s_{4}$ from these randomly generated configurations coincides with that obtained from our simulations. Thus, we can conclude that at ambient pressure, water tends to form a cluster of $S$ states mainly due to random packing rather than a cooperative effect (which would contribute to the enthalpy of mixing $J$ ). This evidence, together with the short correlation length (Sec. IV E 3), suggests that in the temperature and pressure ranges we studied in this paper, far from the hypothesized critical point, even if it exists, the cooperativity effect is indeed negligible.

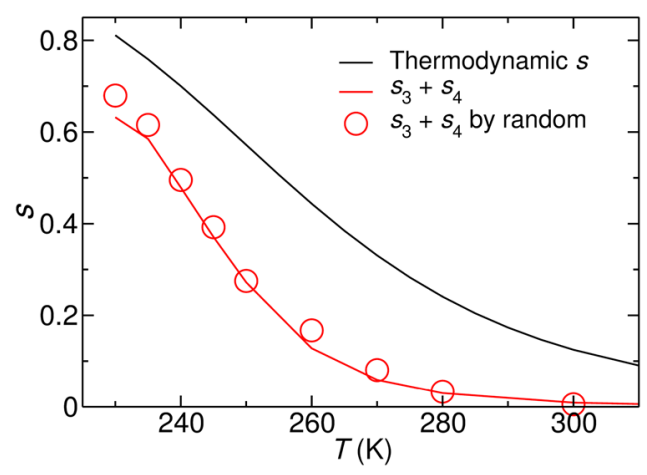

FIG. 12. Fraction of dynamic $S$ states calculated from simulations and random packing of TIP5P water at 1 bar. Solid black and red lines denote fractions of thermodynamic and dynamic $S$ states, respectively. Here the dynamic $S$ state is defined as clustered $S$ states- $S$ water with $3\left(s_{3}\right)$ or $4\left(s_{4}\right) S$ neighbors. Red circles represent fraction of clustered $S$ states estimated from random packing (see text). 
(a)
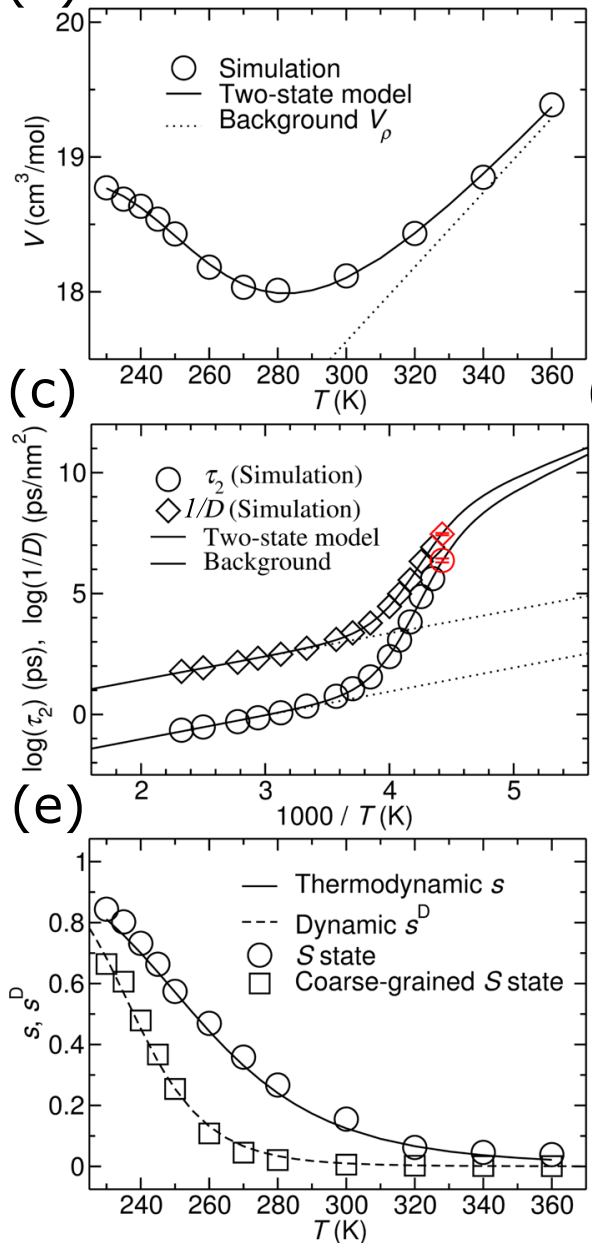

(b)
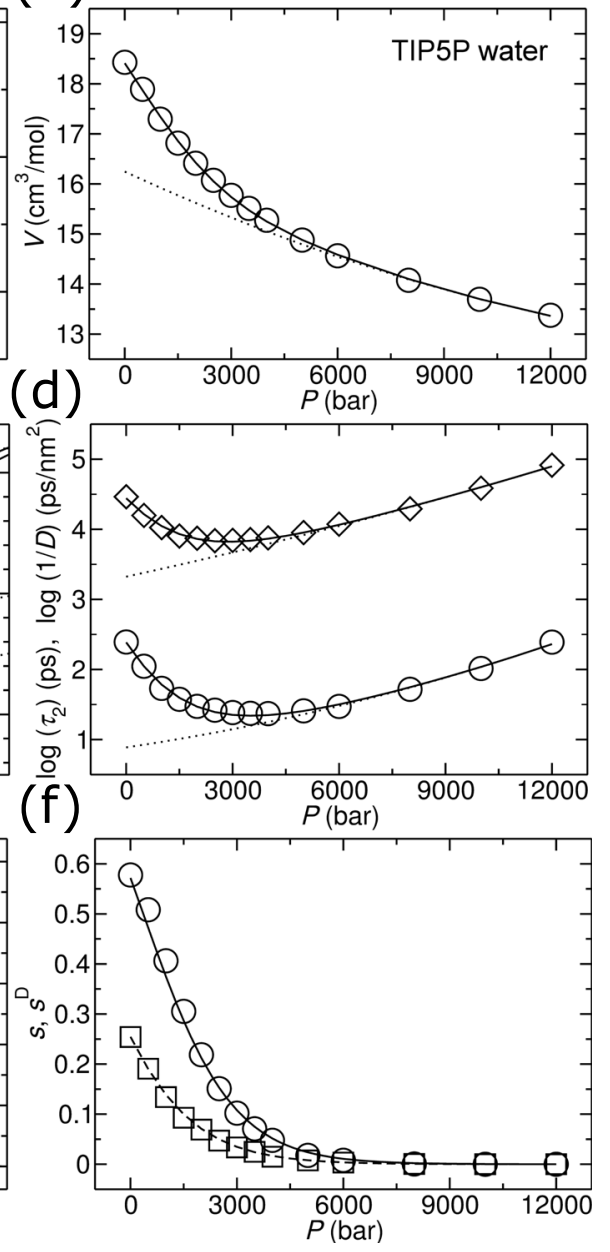

FIG. 13. Water's anomalies in TIP5P water at 1 bar [(a), (c), and (e)] and at $250 \mathrm{~K}$ [(b), (d), and (f)]. [(a) and (b)] Specific volume as a function of temperature and pressure, respectively. [(c) and (d)] Reorientational time $\left(\tau_{2}\right)$ and inverse diffusion coefficient $(1 / D)$ in $\log$ scale as a function of temperature and pressure, respectively (the error bars at $226 \mathrm{~K}$ calculated from three independent $11 \mu$ s trajectories are shown by red color). In (a)-(d), dotted lines represent the normal behaviors of the $\rho$ state. [(e) and (f)] Fractions of thermodynamic and dynamic $S$ states as a function of temperature and pressure, respectively. The fractions of thermodynamic and dynamic $S$ states were obtained from the original and coarsegrained $\zeta$, respectively, from microscopic structures. Solid and dashed lines are the two-state fits to thermodynamic and dynamic order parameters, respectively, which are also used to describe the data in (a)-(d). Panel (c) is reproduced with permission from Shi et al., Proc. Natl. Acad. Sci. U. S. A. 115, 9444-9449 (2018). Copyright 2018 Proceedings of the National Academy of Sciences, USA.

\section{Common structural origin of water's anomalies}

\section{Thermodynamic anomalies of TIP5P water}

Figures 13(a) and 13(b) plot the temperature and pressure dependences of specific volume (circle) together with the two-state fits (solid line) at 1 bar and $250 \mathrm{~K}$, respectively, for TIP5P water. The two-state model (see Sec. II) well describes the simulation data and provides a simple explanation of the density anomaly: at high temperatures, the $\rho$ state dominates and thus the volume decreases upon cooling as normal liquids [the dotted line in Fig. 13(a)]; upon further cooling, the $S$ state with a larger specific volume grows rapidly, thus causing the density maximum. This is a direct consequence of high correlation between $\zeta$ and the local volume [see Figs. 4(b) and 4(c)]. The two-state fitting parameters, listed in Table II, show that the $S$ state has lower energy, smaller entropy, and larger volume than the $\rho$ state, in agreement with our analysis based on the microscopic structural descriptor $\zeta$ (see Sec. IV A).

\section{Dynamic anomalies of TIP5P water}

In Figs. 13(c) and 13(d), we show that water's reorientational time $\left(\tau_{2}\right)$ and inverse diffusion coefficient $(1 / D)$ can also be well described by Eq. (2). Here we stress that the $T$, $P$-dependence of $s$ is independently estimated and supported by structural analysis (see Fig. 10), whose results are shown in Figs. 13(e) and 13(f). Again, the dynamic $\rho$ state behaves normally (see dotted lines). In Fig. 13(c), we demonstrate that water's dynamical (both translational and rotational) slowing down upon cooling can be explained by the hierarchical twostate model without introducing any divergence or glassiness. Equation (2) tells us that the dynamics should show crossovers from the high-temperature Arrhenius behavior with the activation energy $E_{\mathrm{a}}^{\rho, X}$, where $s^{\mathrm{D}} \sim 0$, to the low-temperature one with the activation energy $E_{\mathrm{a}}^{S, X}$, where $s^{\mathrm{D}} \sim 1$, through the two-state regime. As shown in Fig. 13(c), both $1 / D$ and $\tau_{2}$ indeed show such crossover behaviors. On the other hand, the dynamic anomaly as a function of pressure shown in Fig. 13(d) can be interpreted as the reduction of the dynamic $S$ state, with

TABLE II. Two-state parameters for TIP5P water.

\begin{tabular}{|c|c|c|c|c|}
\hline & & \multicolumn{3}{|c|}{ Dynamic } \\
\hline \multicolumn{2}{|c|}{ Thermodynamic (static) } & & Rotation & Translation \\
\hline$\Delta E(\mathrm{~K})$ & -3356 & $\Delta E^{D}(\mathrm{~K})$ & \multicolumn{2}{|c|}{-5298} \\
\hline$\Delta \sigma$ & -13.1 & $\Delta \sigma^{D}$ & \multicolumn{2}{|c|}{-22.3} \\
\hline$\Delta V\left(\mathrm{~cm}^{3} / \mathrm{mol}\right)$ & 16.6 & $\Delta V^{D}\left(\mathrm{~cm}^{3} / \mathrm{mol}\right)$ & \multicolumn{2}{|c|}{15.6} \\
\hline$n$ & 4.38 & $\Delta E_{a}(\mathrm{~K})$ & 3388 & 2530 \\
\hline \multirow{2}{*}{$T_{s=\frac{1}{2}}$} & 255.5 & $E_{a}^{\rho}(\mathrm{K})$ & 2267 & 2219 \\
\hline & & $T_{s^{\mathrm{D}}=\frac{1}{2}}$ & \multicolumn{2}{|c|}{237.6} \\
\hline
\end{tabular}


a larger volume and a higher activation energy, upon compression. Here we note that this two-state feature in dynamics is consistent with a recent experimental finding of the presence of two distinct local structures from measurements of water intermolecular vibrations and the structural relaxation process. ${ }^{65}$

\section{T, P-dependence of the order parameters}

Now we discuss the temperature and pressure dependences of the order parameters $s$ and $s^{\mathrm{D}}$ [Figs. 13(e) and 13(f)]. Thermodynamic $s$ (circle) can be obtained from a twoGaussian fitting of the $\zeta$ distribution (Fig. 3), which agrees well with the result of the two-state fitting to the volume anomalies [Figs. 13(a) and 13(b), solid lines]. To describe dynamic properties, as we mentioned above, we use a coarse-grained structural descriptor $\zeta^{\mathrm{CG}}$ defined as the mean $\zeta$ value within the first shell and define the dynamic $S$ state as those states for which $\zeta^{\mathrm{CG}}>\zeta_{\mathrm{c}}^{\mathrm{CG}}$ (Fig. 9). By using this definition, as shown in Figs. 13(e) and 13(f), we calculated the fraction of the dynamic $S$ state, $s^{\mathrm{D}}$ (square), directly from microscopic structures. We find that it is in excellent agreement with the result independently estimated by two-state fitting to dynamic anomalies (broken line). This clearly shows the validity of the hierarchical two-state model. Similar to the interpretation of the Widom line as the $s=\frac{1}{2}$ line (in the two-state model language, the Schottky anomaly line) near which the thermodynamic response functions maximize, ${ }^{46}$ we propose a $s^{\mathrm{D}}=\frac{1}{2}$ line where the maximization of dynamic fluctuations is expected. $T_{S^{\mathrm{D}}=\frac{1}{2}}$ is approximately $20 \mathrm{~K}$ lower than $T_{s=\frac{1}{2}}$, indicating the crucial role of coarse-graining in bridging the thermodynamic and dynamic anomalies and inferring a significantly different nature of thermodynamic and dynamic quantities: the former (linked to static structures) can be determined locally as long as there is little cooperativity, whereas the latter (defined by the movement of molecules) cannot be estimated locally because of its intrinsic coupling to the motion of neighboring molecules.

\section{Water's anomalies in ST2 water}

In order to test the validity of our hierarchical two-state scenario, all the calculations were repeated on another widely used water model - the ST2 model (see also Appendix B). All the behaviors shown above can also be seen and described by the hierarchical two-state model in ST2 water (see Fig. 14). (a)
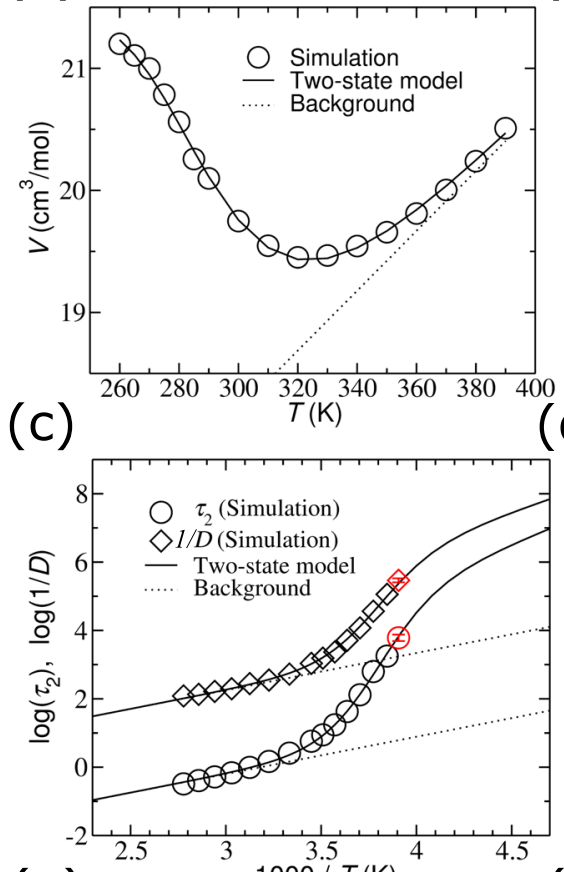

(e)

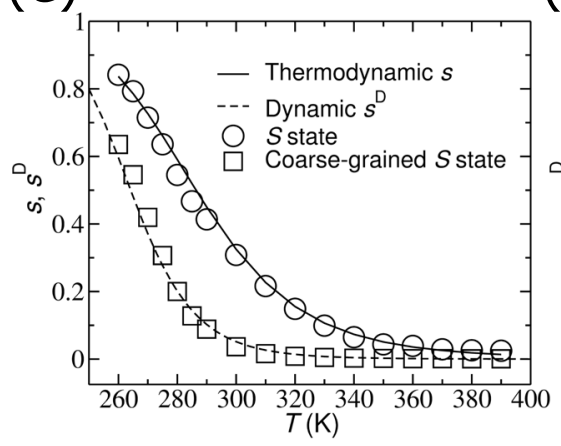

(b)
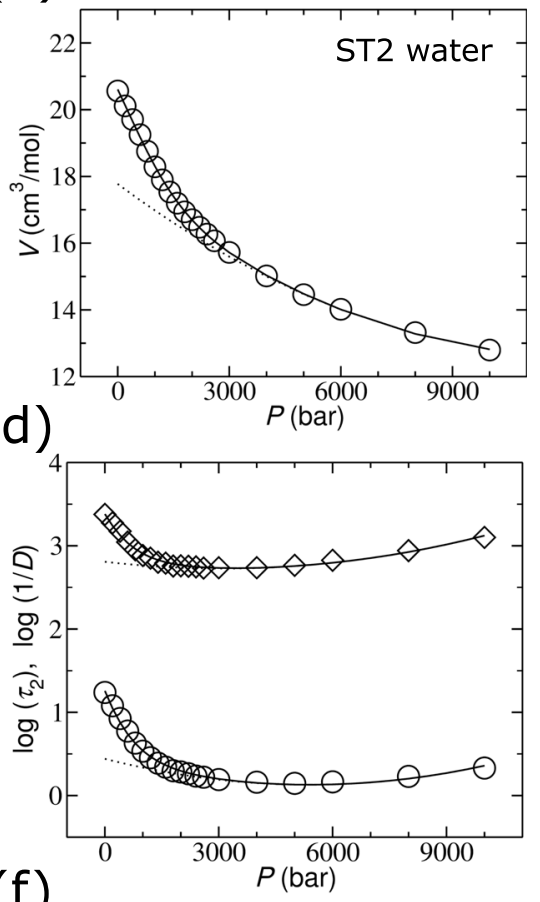

(f)

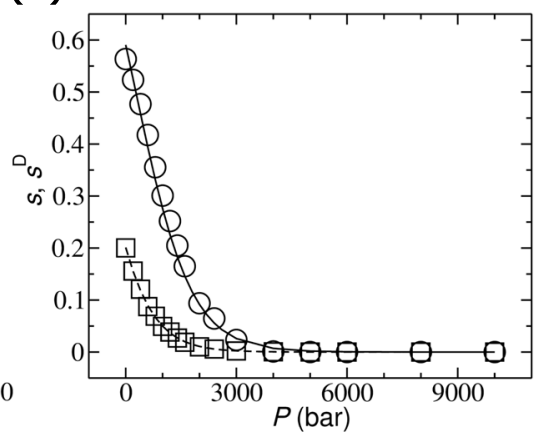

FIG. 14. Water's anomalies in ST2 water at 1 bar $[(a),(c)$, and (e)] and at $280 \mathrm{~K}$ [(b), (d), and (f)]. [(a) and (b)] Specific volume as a function of temperature and pressure, respectively. [(c) and (d)] Reorientational time and inverse diffusion coefficient in log scale as a function of temperature and pressure, respectively (the error bars at the lowest temperature are shown by red color). [(e) and (f)] Fractions of thermodynamic and dynamic $S$ states as a function of temperature and pressure, respectively. Panel (c) is reproduced with permission from Shi et al., Proc. Natl. Acad. Sci. U. S. A. 115, 9444-9449 (2018). Copyright 2018 Proceedings of the National Academy of Sciences, USA. 

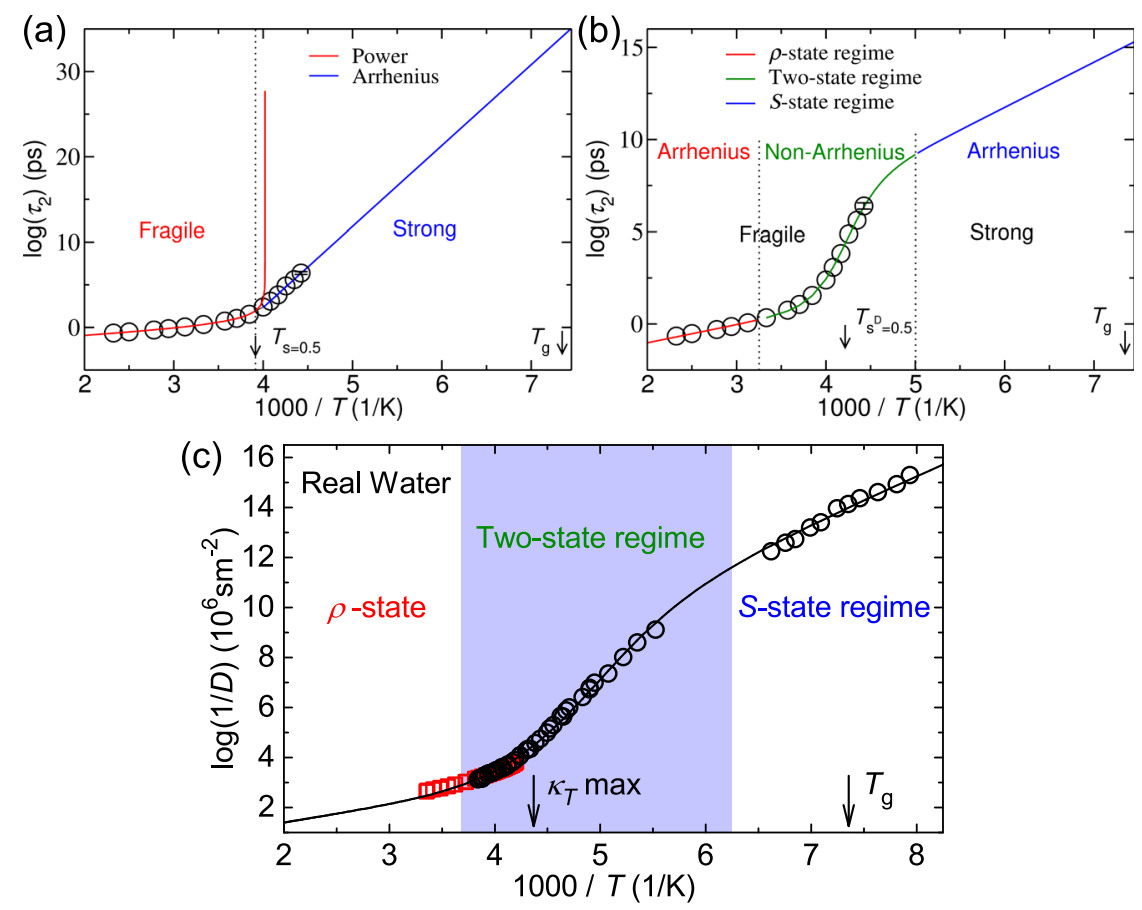

FIG. 15. Dynamical crossover in TIP5P water and real water. (a) A Widom line interpretation of dynamical crossover in TIP5P water: a crossover from the power law above the Widom line ( $T_{s=\frac{1}{2}}$ line here) to Arrhenius law below the Widom line. (b) Hierarchical two-state scenario for the dynamical crossover in TIP5P water: In the dynamic $\rho$-state regime, water has Arrhenius behavior; when entering the two-state regime, water shows non-Arrhenius (apparently "fragile") behavior; in the dynamic $S$-state regime, water exhibits Arrhenius (the so-called "strong") behavior. (c) Two-state fitting to the temperature dependence of the diffusion constant. Red square and black circles are taken from Refs. 81 and 82, respectively. The $\kappa_{T}$ maximum and glass transition temperature are taken from Refs. 12 and 83, respectively. The solid curve is the curve fitted by Eqs. (1) and (2). The fitting parameters are determined as $E_{a}^{\rho, D}=1567 \mathrm{~K}, \Delta E_{a}^{D}=2813 \mathrm{~K}$, $\Delta E^{D}=-2191 \mathrm{~K}$, and $\Delta \sigma^{D}=-10.5 \mathrm{~K}$. From the fitting result, we predict $T_{s} \mathrm{D}=0.5=208 \mathrm{~K}$, which is $\sim 20 \mathrm{~K}$ lower than $\kappa_{T}$ maximum temperature, ${ }^{12}$ in agreement with TIP5P and ST2 water. The diffusion coefficients were calculated at 1 bar and measured at atmospheric pressure (red square) and under vacuum (black circle).

The success of our hierarchical two-state scenario in describing both the thermodynamic and dynamic anomalies in the two popular water models as well as real water [see below and Fig. 15(c)] strongly suggests the generality and validity of our hierarchical two-state scenario. We list the two-state parameters for ST2 water in Table III.

\section{Crossover between the two states and the apparent "fragile-to-strong" transition}

Although many experimental measurements support a power-law behavior of viscosity, ${ }^{72,73}$ diffusion, ${ }^{72,73}$ and relaxation time $e^{15,16,72}$ of liquid water above the homogeneous nucleation temperature at low pressure, the calculated

TABLE III. Two-state parameters for ST2 water.

\begin{tabular}{|c|c|c|c|c|}
\hline & & \multicolumn{3}{|c|}{ Dynamic } \\
\hline \multicolumn{2}{|c|}{ Thermodynamic (static) } & & Rotation & Translation \\
\hline$\Delta E(\mathrm{~K})$ & -4613 & $\Delta E^{D}(\mathrm{~K})$ & \multicolumn{2}{|c|}{-6444} \\
\hline$\Delta \sigma$ & -16.1 & $\Delta \sigma^{D}$ & \multicolumn{2}{|c|}{-24.4} \\
\hline$\Delta V\left(\mathrm{~cm}^{3} / \mathrm{mol}\right)$ & 31.0 & $\Delta V^{D}\left(\mathrm{~cm}^{3} / \mathrm{mol}\right)$ & \multicolumn{2}{|c|}{36.6} \\
\hline$n$ & 6.5 & $\Delta E_{a}(\mathrm{~K})$ & 2615 & 1837 \\
\hline \multirow{2}{*}{$T_{s=\frac{1}{2}}$} & 286.5 & $E_{a}^{\rho}(\mathrm{K})$ & 2510 & 2510 \\
\hline & & $T_{s^{\mathrm{D}}=\frac{1}{2}}$ & \multicolumn{2}{|c|}{264.1} \\
\hline
\end{tabular}

rotational relaxation time and diffusion constant from our simulations in a much wider temperature range fail to follow the power law, in agreement with other simulation results (see, for example, Refs. 8, 27, 28, 74, and 75). On the other hand, many pieces of evidence supporting the Arrhenius behavior of water near $T_{\mathrm{g}}$ have been consistently found in confined, ${ }^{9,76}$ lowdensity amorphous, ${ }^{77,78}$ high-density amorphous, ${ }^{78}$ and vapordeposited amorphous water. ${ }^{77}$ A "fragile-to-strong" transition below the homogeneous nucleation temperature was proposed to interpret this unusual dynamic behavior of water. ${ }^{21,79,80} \mathrm{It}$ ascribes water's unusual dynamics to either the glass transition or the second critical point. The former, based on either MCT $^{17-19,27,74}$ or Adam-Gibbs theory, ${ }^{21,80}$ can hardly explain why the dynamical slowing down starts so far from $T_{\mathrm{g}}$ [see Fig. 15(c)]. The latter, as shown in Fig. 15(a), interprets water's unusual dynamics as a crossover from a power-law to Arrhenius behavior at the Widom line ( $T_{s=\frac{1}{2}}$ line here). ${ }^{8,27,28}$ This interpretation, although consistent with the dynamical slowing down far from $T_{\mathrm{g}}$, suffers from three fundamental difficulties: (1) the experimental power-law divergence temperature $T_{\mathrm{MCT}}$ $\simeq 1.6 T_{\mathrm{g}}{ }^{73}$ does not obey the empirical rule $T_{\mathrm{MCT}} \simeq 1.2 T_{\mathrm{g}}$ found in many other glass-forming liquids; (2) the large difference between $T_{\mathrm{W}}$ and $T_{\mathrm{g}}$ leads to unrealistic prediction of either a too long relaxation time at $T_{\mathrm{g}}=136 \mathrm{~K}$ or a too high $T_{\mathrm{g}}\left(T_{\mathrm{g}} / T_{\mathrm{m}}\right.$ $\simeq 0.65-0.7$ ) in water models [see Fig. 15(a)]; (3) the Widom line scenario cannot explain the new experimental diffusion data below $T_{\mathrm{W}}$ [see Fig. 15(c)]. It is worth noting that even 
though MCT predicts an unusually high value of $T_{\mathrm{MCT}} / T_{\mathrm{g}}$, it correctly describes the two-step decay of the intermediate scattering function and the onset of dynamic slowing down of liquid water. ${ }^{17-19,27,74}$

On the other hand, our hierarchical two-state model predicts a dynamic crossover between two regimes of Arrhenius behavior, centered at the $s^{\mathrm{D}}=\frac{1}{2}$ line, as shown in Figs. 13(c), 14(c), and 15(b). There we can see that our hierarchical twostate model not only quantitatively describes our simulation results over a wide temperature range but also predicts a reasonable rotational time scale (1000 s for TIP5P and $10 \mathrm{~s}$ for ST2) at the glass transition temperature $T_{\mathrm{g}}=136 \mathrm{~K}^{83}$

Moreover, recent measurements on bulk water ${ }^{82}$ and previous experiments on confined water, ${ }^{22,24,26}$ both showed an accelerated dynamic slowing down [convex at $T \sim 250 \mathrm{~K}$ in Fig. 15(b)], followed by a decelerated one [concave at $T \sim 210 \mathrm{~K}$ in Fig. 15(b)] upon cooling. As shown in Fig. 15(c), the hierarchical two-state model almost perfectly describes this unique (convex-to-concave) temperature dependence of the diffusion constant measured experimentally, ${ }^{81,82}$ which cannot be explained by other scenarios. Furthermore, the fragile-to-strong transition should be associated with glass transition by definition, but it takes place in a temperature region too far away from the glass transition temperature $T_{\mathrm{g}}$ $\sim 136 \mathrm{~K}$. These facts cast a strong doubt on the validity of this interpretation.

Here, we propose an alternative explanation based on the hierarchical two-state model: a structural crossover from the dynamic $\rho$ state to $S$ state region leads to a dynamic crossover between two Arrhenius behaviors, apparently emergent as a "fragile-to-strong" transition ${ }^{21,79,80}$ [see Figs. 15(b) and 15(c)]. In the hierarchical two-state scenario, liquid water in the dynamic $\rho$-state regime has Arrhenius behavior; the "apparent" fragile behavior of supercooled liquid water originates from the increasing weight of a more viscous dynamic $S$ state in a two-state mixture; further cooling into the dynamic $S$ state dominating regime leads to the saturation of the order parameter $s^{\mathrm{D}}\left(s^{\mathrm{D}} \rightarrow 1\right)$, resulting in the deceleration of the dynamic slowing down and the strong liquid (Arrhenius) behavior.

Here we stress that this apparent fragile-to-strong transition cannot be described by normal glassy behavior linked to glass transition and is a direct consequence of the twostate behavior. We speculate that there may be a similar two-state feature originating from the formation of locally favored structures behind "apparent" fragile-strong transitions in other glass-forming liquids such as silica (see, e.g., Refs. 84 and 85) and metallic glass-formers (see, e.g., Ref. 86). When entering and leaving the two-state mixture regime, centered at the $s^{\mathrm{D}}=\frac{1}{2}$ line $\left(T_{s^{\mathrm{D}}=\frac{1}{2}}=237.6 \mathrm{~K}\right.$ for TIP5P, $264.1 \mathrm{~K}$ for ST2 water and $208 \mathrm{~K}$ for real water at ambient pressure), an Arrhenius-to-Arrhenius dynamic crossover, known as an emergent fragile-to-strong transition, is predicted by the two-state scenario, ${ }^{34}$ consistent with experimental observations in confined $22,24,26$ and bulk ${ }^{82}$ water. Caupin and co-workers ${ }^{40,42}$ have recently proposed a different two-state model by assuming a $\rho$ state that follows VogelFulcher-Tammann (VFT) instead of Arrhenius behavior. This analysis is based on the presence of a critical point at low pressure $\left(T_{c}=228.2 \mathrm{~K} \text { and } P_{c}=0 \mathrm{MPa}\right)^{87}$ and a divergence of the dynamics (e.g., $1 / D$ ) at a finite temperature ( $T=147.75 \mathrm{~K})$. These assumptions are, however, inconsistent with recent experimental measurements and simulation results that found neither a low-pressure critical point ${ }^{10,11,51,88-90}$ nor divergent dynamics. ${ }^{77,78,82}$ Our model, which is built from microscopic information, instead supports Arrhenius behaviors of both $\rho$ and $S$ states, in agreement with the data reported experimentally $^{82}$ [see Fig. 15(c)].

\section{E. Dynamic heterogeneities as a two-state feature}

We will now show that strong evidence for the hierarchical two-state scenario comes from the study of the apparent glassy behaviors of water (see Appendix A).

\section{Discovery of the unusual dynamic heterogeneity maximum}

We first focus on the translational and rotational four-point susceptibility $\chi_{4}^{\mathrm{T}, \mathrm{R}}(t)$ characterizing the fluctuations of translational and rotational dynamics around the average, respectively [Eq. (A1) in Appendix A]. $\chi_{4}^{\mathrm{T}, \mathrm{R}}(t)$ has a distinct maximum at a dynamic time scale $\tau_{4}$ (Figs. 22, 23, 26, and 27). For normal glass-forming liquids, $\chi_{4}^{\mathrm{T}, \mathrm{R}}\left(\tau_{4}\right)$ usually increases monotonically as approaching the glass transition temperature $T_{\mathrm{g}}$. Contrary to this glass phenomenology, for both TIP5P and ST2 water, it has a distinct peak in the narrow dynamic Schottky (DS) band ${ }^{29}$ (green region) where $s^{\mathrm{D}} \simeq \frac{1}{2}$ (Fig. 16), similar to the maximization of the thermodynamic response functions near the Widom (or Schottky) line. ${ }^{8,46}$ The maximization of dynamic heterogeneity can also be seen from the behavior of the stretching parameter $\beta$ [Eq. (5)], which describes the deviation of molecular dipole reorientation from a Debye process $(\beta=1)$. Non-Debye behavior $(\beta<1)$ is usually attributed to heterogeneous dynamics. Figure 16 shows that $\beta$ reaches a minimum at $T_{s^{\mathrm{D}}=\frac{1}{2}}$, again confirming the maximization of dynamic heterogeneity in the DS band.

We argue that this is a unique feature of the two-state model, which we call the Schottky anomaly. ${ }^{30,46}$ The fact that maximization of translational and orientational four-point susceptibilities and minimization of the stretching parameter occur in the narrow DS band where $s^{\mathrm{D}} \simeq \frac{1}{2}$ strongly supports the two-state behavior of supercooled water. By running several independent long-time simulations, we confirmed that the maximization of $\chi_{4}^{\mathrm{T}, \mathrm{R}}\left(\tau_{4}\right)$ is not due to statistical errors, which can be seen from the error bars shown in Fig. 16. The coincidence of maximization of $\chi_{4}^{\mathrm{T}, \mathrm{R}}(t)\left(\tau_{4}\right)$ and minimization of $\beta$ at $T_{s^{\mathrm{D}}=\frac{1}{2}}$ in both models, indicative of the two-state scenario, strongly supports the observations.

Despite very long time simulation (33 $\mu$ s for the TIP5P model), due to the significant slowdown of dynamics, the data at the lowest temperature still suffer from large statistical fluctuations. The increase of $\chi_{4}^{\mathrm{T}, \mathrm{R}}\left(\tau_{4}\right)$ at the lowest temperature (226 K for TIP5P and $256 \mathrm{~K}$ for ST2 water) may come from a general tendency of growth of dynamic heterogeneity with decreasing temperature. ${ }^{91}$

Finally we stress that this feature cannot be explained by the scenario based on glass transition, where $\chi_{4}^{\mathrm{T}, \mathrm{R}}(t)$ should increase monotonically when approaching $T_{\mathrm{g}}$, and thus in 

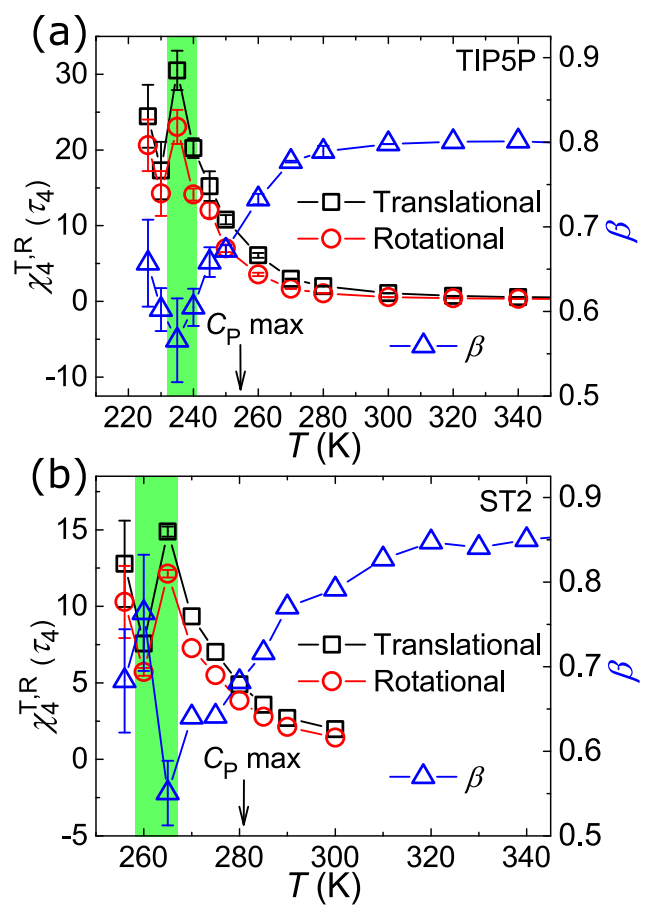

FIG. 16. Dynamic heterogeneities in (a) TIP5P and (b) ST2 water at 1 bar. Both translational (black squares) and rotational (red circles) four-point susceptibilities $\chi_{4}^{\mathrm{T}, \mathrm{R}}\left(\tau_{4}\right)$ [Eq. (A1)] exhibit a peak in the dynamic Schottky (DS) band (green region) where $s^{\mathrm{D}} \simeq \frac{1}{2}$ [see Figs. 13(e) and 14(e)], providing strong evidence of the two-state behavior. The stretching parameter $\beta$ (blue triangles), which is determined by fitting the stretched exponential function [Eq. (5)] to the second Legendre polynomial of the time correlation function of molecular dipole moment [Eq. (4)], also shows a minimum appearing around $T_{s^{\mathrm{D}} \simeq \frac{1}{2}}$. These results confirm the maximization of dynamic heterogeneities in the DS band, strongly supporting the two-state picture. Reproduced with permission from Fig. 3 of Shi et al., Proc. Natl. Acad. Sci. U. S. A. 115, 9444-9449 (2018). Copyright 2018 Proceedings of the National Academy of Sciences, USA.

principle, no $\chi_{4}^{\mathrm{T}, \mathrm{R}}(t)$ maximization should occur above $T_{\mathrm{g}}$. Furthermore, the absence of the peak at the static Widom line indicates that there is little critical anomaly associated with the Widom line since, if it exists, critical fluctuations should be maximized there, which should give rise to the dynamical heterogeneity maximum on the Widom line. Thus, the maximum of dynamic heterogeneity below the Widom line and far above the glass transition supports our hierarchical two-state model and runs against the scenarios based on the criticality associated with the Widom line and the glass transition at least at ambient pressure.

\section{The violation of the Stokes-Einstein-Debye relation caused by the two-state feature}

In normal glass-forming liquids, rotational motion decouples from translational diffusion below approximately $1.2 T_{\mathrm{g}},{ }^{3}$ well known as the breakdown of the Stokes-Einstein and Stokes-Einstein-Debye relations $\left(D \tau_{2}=2 a^{2} / 9\right.$, where $a$ is an effective hydrodynamic radius). This is a consequence of glassy dynamic heterogeneity. However, as shown in Fig. 17, the breakdown happens much earlier $\left(\sim 2 T_{\mathrm{g}}\right)$ than $1.2 T_{\mathrm{g}}$, given that the melting point of the TIP5P model is very close to real water. Here we show that this unusual behavior can also be naturally explained by the hierarchical two-state scenario.
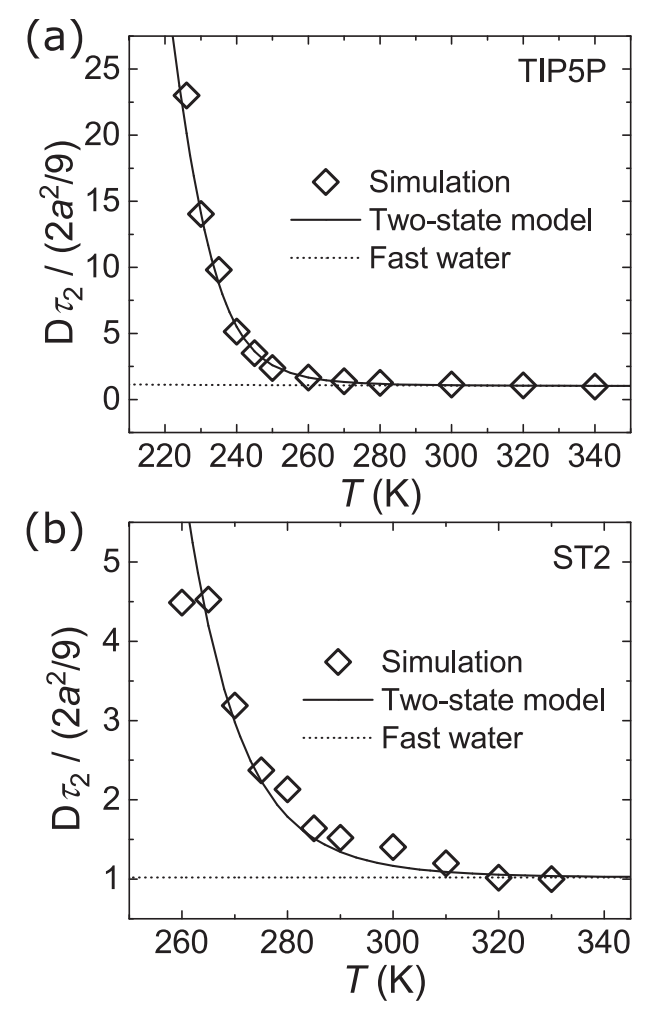

FIG. 17. Breakdown of the Stokes-Einstein-Debye relation in (a) TIP5P and (b) ST2 water at 1 bar. Solid and dotted lines represent the two-state and individual fast-water (dynamic $\rho$ state) contributions, respectively, indicating that the growth of the fraction of slow water (dynamic $S$ state) upon cooling results in the breakdown of the Stokes-Einstein-Debye relation in supercooled water. The effective hydrodynamic radius $a=1.3 \AA$ and $1.2 \AA$ was estimated from the high temperature data for TIP5P and ST2 water, respectively. Reproduced with permission from Fig. 4 of Shi et al., Proc. Natl. Acad. Sci. U. S. A. 115, 9444-9449 (2018). Copyright 2018 Proceedings of the National Academy of Sciences, USA.

Applied to diffusive and rotational motions, Eq. (2) naturally gives a reasonable explanation to the Stokes-Einstein-Debye relation by

$$
\begin{aligned}
D \tau_{2} & =D_{0} \tau_{0} \exp \left[\frac{E_{\mathrm{a}}^{\rho, \tau}-E_{\mathrm{a}}^{\rho, D}}{k_{\mathrm{B}} T}\right] \cdot \exp \left[\frac{\Delta E_{\mathrm{a}}^{\tau}-\Delta E_{\mathrm{a}}^{D}}{k_{\mathrm{B}} T} \cdot s^{\mathrm{D}}\right] \\
& \approx D_{0} \tau_{0} \exp \left[\frac{\Delta E_{\mathrm{a}}^{\tau}-\Delta E_{\mathrm{a}}^{D}}{k_{\mathrm{B}} T} \cdot s^{\mathrm{D}}\right],
\end{aligned}
$$

where $D$ is the diffusion constant and $\tau_{2}$ is the reorientational time. The second equation is valid only if the activation energies for rotation and diffusion equal in the dynamic $\rho$ state $\left(E_{\mathrm{a}}^{\rho, \tau} \approx E_{\mathrm{a}}^{\rho, D}\right)$, i.e., rotation is coupled to diffusion. This is true since the Stokes-Einstein-Debye relation holds at high temperatures, where $s^{\mathrm{D}} \sim 0$. This is also confirmed by our fitting result (Tables II and III). At high temperatures, the dynamic $\rho$ state predominates and, as a result, translational motion couples to reorientation very well. However, the activation energy for reorientation is considerably higher than translation in the dynamic $S$ state (Tables II and III), so the reorientation will slow down much faster than translation upon cooling, which leads to the breakdown of the Stokes-Einstein-Debye relation [see Eq. (6)]. It can be seen clearly in Fig. 17 that the $\rho$ state follows the Stokes-Einstein-Debye relation quite well and the decoupling behavior can be perfectly described by the 
prediction of the hierarchical two-state model [Eq. (6)], strongly indicating that the anomalous breakdown mainly comes from the growth of the dynamic $S$ state upon cooling and not from the glassiness. Here we stress that no more fitting parameters are involved in the comparison of Eq. (6) and the data in Fig. 17.

\section{Dynamic correlation length}

The spatial correlation of dynamic heterogeneity can be described by ${ }^{92}$

$$
\begin{aligned}
g_{4}(r)= & \left\langle\frac{1}{4 \pi r^{2} N \rho} \sum_{i \neq j}^{N} \delta\left(r-\left|\boldsymbol{r}_{i}(0)-\boldsymbol{r}_{j}(0)\right|\right) w_{i}^{\mathrm{T}}(t) w_{j}^{\mathrm{T}}(t)\right\rangle \\
& -\left\langle\frac{Q(t)}{N}\right\rangle^{2},
\end{aligned}
$$

where $\rho$ is the number density, $N$ is the number of particles in the system, and $\boldsymbol{r}_{i}(0)$ is the position of particle $i$ at time 0 . The definition of the overlap function $w_{i}^{\mathrm{T}}(t)$ is given in Appendix A. Then, the dynamic correlation length $\xi_{4}$ can be extracted by an envelope fit of $g_{4}(r)$ to an exponential function,

$$
f(r)=f_{0} \exp \left(-\frac{r}{\xi_{4}}\right),
$$

where $f_{0}$ is a constant. In the envelope fit, the first two peaks of $g_{4}(r)$ were not included because they may be affected by strong translational ordering. In Fig. 18, we show the correlation function $g_{4}(r)$ as a solid curve and its envelope fit with a dashed line, from which the correlation length can be obtained. As shown in Fig. 19, the correlation length $\xi_{4}(T)$ maximum is $\sim 6 \AA$ at $T_{S^{\mathrm{D}}=\frac{1}{2}}$ in the DS band, corresponding to 2 molecular size, which agrees well with a short structural correlation length of $\sim 4 \AA$ recently reported by femtosecond x-ray experiment. ${ }^{12}$ The short correlation length suggests little cooperativity or criticality in liquid water at ambient pressure, in agreement with our above discussions in Sec. IV B 6. It also confirms that the finite size effects, if any, should be negligible in our systems.

In summary, the four-point susceptibility, stretching parameter, and Stokes-Einstein-Debye relation all together provide strong evidence for the two-state scenario, while they run against the glass transition scenario and the scenario based

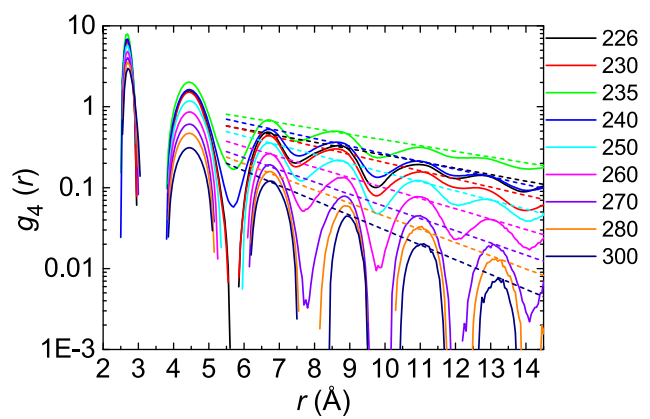

FIG. 18. Spatial correlation function $g_{4}(r)$ at the time scale of $\tau_{4}$ in TIP5P water at 1 bar. The dashed line is an envelope fit to an exponential function. The first two peaks were not included in the fit. Reproduced with permission from Fig. S12 of Shi et al., Proc. Natl. Acad. Sci. U. S. A. 115, 9444-9449 (2018). Copyright 2018 Proceedings of the National Academy of Sciences, USA.

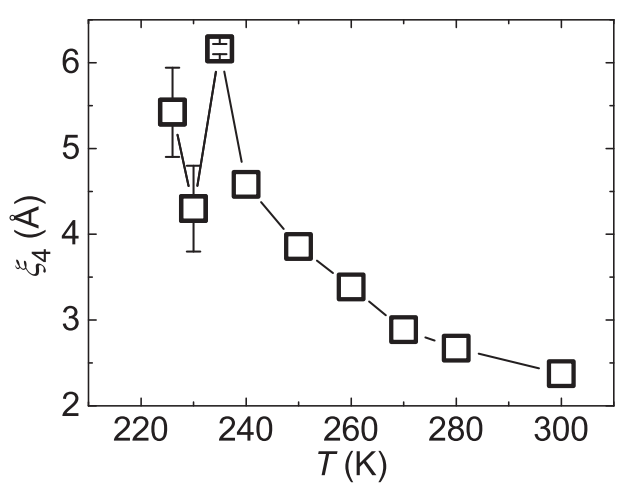

FIG. 19. Dynamic correlation length $\xi_{4}$ as a function of temperature for TIP5P water at 1 bar. The error bar shows the standard error of $\xi_{4}$ calculated from 3, 10, and 4 independent microsecond-long trajectories at 226, 230, and $235 \mathrm{~K}$, respectively (see Table I for details). Reproduced with permission from Fig. S13 of Shi et al., Proc. Natl. Acad. Sci. U. S. A. 115, 9444-9449 (2018). Copyright 2018 Proceedings of the National Academy of Sciences, USA.

on criticality associated with the Widom line. The two-state scenario, along with the microscopic structural descriptor $\zeta^{\mathrm{CG}}$, taking into account first-shell environment by coarse graining can instead well explain the dynamic heterogeneity in water. The dynamic heterogeneity of water in the $T-P$ region studied here is simply a consequence of the dynamical coexistence of $\rho$ and $S$ states [see Figs. 4(d)-4(f)].

\section{DISCUSSION}

Until now, we have demonstrated that the hierarchical two-state scenario can explain both thermodynamic and dynamic anomalies of water, as well as its dynamic heterogeneities, in a coherent manner. From a structural point of view, it provides an excellent description of the anomalies in the region where critical effects are negligible. Under ordinary conditions, far from the critical point, criticality is expected to have negligible effects (e.g., $\frac{T_{\rho \max }}{T_{\mathrm{C}^{\prime}}}-1=0.3$ and $\left.\frac{P_{\text {atm }}}{P_{\mathrm{C}^{\prime}}}-1=-0.9997\right)$. The absence of the effect of criticality on water's anomalies in the $T-P$ range we studied is supported by the absence of cooperativity in the formation of locally favored structures (see Fig. 12), the short correlation length (see Fig. 19), and the almost perfect fitting of the $T, P$-dependence of $s$ by Eq. (1) without cooperativity [see Figs. 13(e) and 13(f) and Figs. 14(e) and 14(f)].

The hierarchical two-state model incorporates dynamical effects in a purely static thermodynamic framework by considering the activation barrier for motion and its link to the coarse-grained structural order parameter. It explains the dynamic anomaly as a continuous crossover from $\rho$-rich to $S$-rich water upon cooling. Since this crossover depends only on the fraction of the dynamic $S$ state, without relying upon glassy slow dynamics, it is different from previous explanations based on the slowing down of dynamics near $T_{\mathrm{g}}$. These conventional scenarios are underpinned by MCT, predicting a power-law divergence of the structural relaxation time (or viscosity) towards $T_{\mathrm{MCT}}$ or by the Adam-Gibbs theory that predicts a VFT behavior. ${ }^{5}$ At ambient pressure, a recent measurement of viscosity in a wide temperature range 239-373 K rules out the VFT behavior and supports a power law with a divergent temperature $T_{\mathrm{MCT}}=226 \mathrm{~K} .{ }^{73}$ However, its location 
does not obey the empirical rule of $T_{\mathrm{MCT}} \sim 1.2 T_{\mathrm{g}}$ established for many glass-formers. On the other hand, MCT well describes the two-step decay of the intermediate scattering function and the onset of non-Arrhenius dynamic behavior of liquid water at low pressure. ${ }^{17-19,27,74}$ MCT is a theory that explains the slow dynamics of supercooled liquid by a structural change in a liquid near $T_{\mathrm{g}}$. So MCT seems to capture the onset of the non-Arrhenius behavior in a weakly supercooled regime by detecting the (non-glassy) structural change of water in the liquid in this temperature region. In relation to this, it is interesting to note that, in this region, our prediction and the power-law prediction are almost indistinguishable in the functional form [see, e.g., Fig. 2(a) in Ref. 34]. At the same time, however, MCT can explain neither the diffusion data in a deeply supercooled region ${ }^{82}$ nor those at $P$ $>1500$ bars. ${ }^{15}$ Furthermore, recent experiments showed the absence of any singularities in water's dynamics at ambient pressure $^{77,78,82}$ [see, e.g., Fig. 15(c)]. Here we note that the diffusion results have been interpreted as a fragile (power law)-to-strong (Arrhenius) transition. In reality, however, the data clearly show an Arrhenius-to-Arrhenius crossover, in agreement with our prediction based on the hierarchical twostate scenario (see Fig. 15). All these pieces of evidence, combined with the dynamic anomalies upon compressing (see Figs. 13 and 14), which are difficult to explain by the glass transition scenario, the maximization of $\chi_{4}^{\mathrm{T}, \mathrm{R}}(t)$ at $T_{s^{\mathrm{D}}=\frac{1}{2}}$ and the breakdown of the Stokes-Einstein-Debye relation far above $1.2 T_{\mathrm{g}}$, clearly suggest that water's dynamic anomalies,

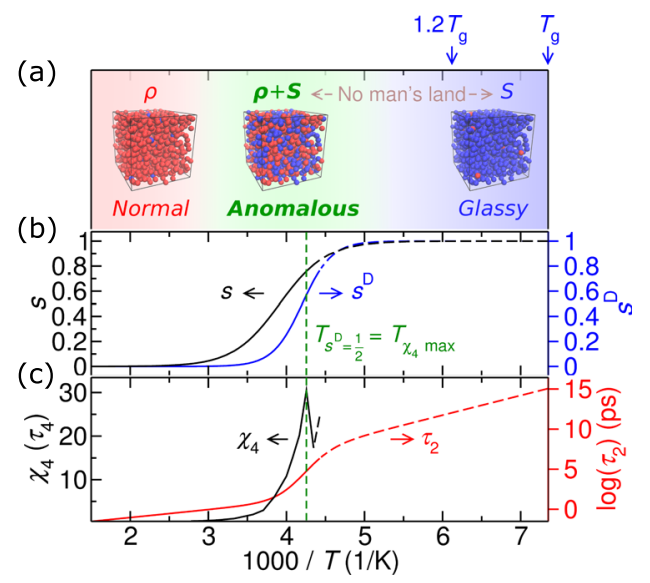

FIG. 20. Hierarchical two-state scenario of water's anomalies. (a) In the twostate scenario, the full temperature range can be divided into three regimes: (1) At high temperatures ( $\rho$ state regime), water is predominated by the disordered $\rho$ state and behaves like normal liquids; (2) in the supercooled regime (two-state regime), water is mainly a mixture of $\rho$ and $S$ states, behaving anomalously; (3) below the two-state regime ( $S$ state regime), water is mostly in the $S$ state and shows glassy behavior typical of strong liquids. (b) Fractions of thermodynamic and dynamic $S$ states as a function of inverse temperature at 1 bar for TIP5P water. (c) Reorientational time $\tau_{2}$ in log scale and four-point susceptibility $\chi_{4}\left(\tau_{4}\right)$ as a function of inverse temperature at 1 bar for TIP5P water. At ambient pressure, dynamic $S$ states grow rapidly upon cooling and their fraction reaches a value of 0.5 at $T_{s^{\mathrm{D}}=\frac{1}{2}}$, where $\chi_{4}\left(\tau_{4}\right)$ maximizes, indicating unique two-state behavior. When entering and leaving the two-state regime, dynamic quantities show an Arrhenius-to-Arrhenius crossover, centered by the $s^{\mathrm{D}}=\frac{1}{2}$ line. The two-state regime is far from the glass transition temperature $T_{\mathrm{g}}$, and as a result, the dynamic slowing down, dynamic heterogeneity, and Stokes-Einstein-Debye relation breakdown all start far away from $T_{\mathrm{g}}$.

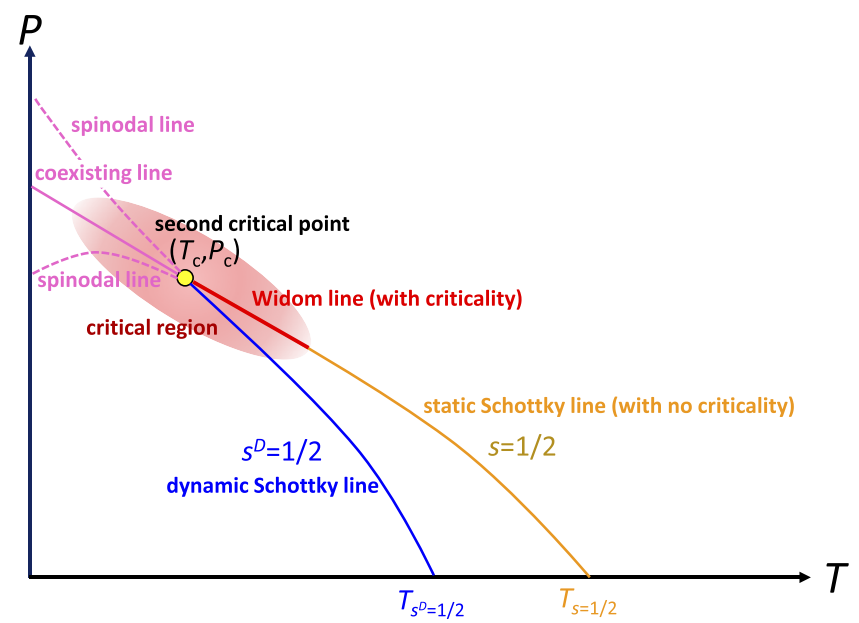

FIG. 21. Schematic figure showing the role of criticality on water's anomalies. The effect of criticality associated with the second critical point (yellow point) at $\left(T_{c}, P_{c}\right)$ may be limited in the critical regime shaded in red color. Outside this region, criticality does not play any important role, and thus, a simple two-state scenario without cooperativity should be valid there. Thus, it is expected that the Widom line with criticality (red solid line) continuously transforms to the (two-state) Schottky line without any criticality (orange solid line). The blue line is the dynamic Schottky line where $s^{\mathrm{D}}(T, P)=1 / 2$. We also draw a coexistence line (pink solid line) of high-density liquid (HDL) and low-density liquid (LDL) as well as spinodal lines (pink dashed lines), which are the stability limit of HDL and LDL.

starting very far from $T_{\mathrm{g}}$, do not originate from the glass transition. On the other hand, our two-state scenario not only naturally explains the apparent "fragile-to-strong" transition and the violation of the Stokes-Einstein-Debye relation far above $1.2 T_{\mathrm{g}}$ but also distinctively predicts the maximization of dynamic fluctuations below the Widom line, in good agreement with experimental and simulation results.

Our hierarchical two-state scenario of water's anomalies is explained in Fig. 20 for TIP5P water. All the anomalies are a consequence of the dynamical coexistence of $\rho$ and $S$ states. We also show the role of the second critical point schematically in Fig. 21. As shown in this figure, we argue that criticality associated with the second critical point is important only in the critical region shaded in red color, where the criticality may induce apparent power-law divergences towards the Widom line. However, outside this critical regime, there is little effect of criticality and the Widom line with criticality transforms continuously to the Schottky line without any criticality.

\section{CONCLUSIONS}

Taken together, the above discussions suggest a unified picture of water's thermodynamic and dynamical anomalies, as illustrated in Fig. 20. At high temperatures ( $\rho$ state regime), water mainly consists of the disordered $\rho$ state and behaves like normal liquids. In a supercooled regime (two-state regime), water contains two local structures with their populations varying rapidly with temperature and pressure. In this two-state regime, water's properties are mainly determined by the hierarchical two-state model. Below the two-state regime and approaching $T_{\mathrm{g}}$ ( $S$ state regime), glassiness should play a more and more important role and eventually predominate water's dynamical behaviors near the glass transition point. Water's thermodynamic and dynamic anomalies locate in the 
two-state regime and therefore originate from the presence of the underlying two distinct local structures.

Water-like dynamic anomalies in the form of fragile-to strong transitions, which were pioneered by Angell and his co-workers, ${ }^{21,23}$ have been seen in many glass-formers such as silica (see, e.g., Refs. 84 and 85), metallic liquids (see, e.g., Refs. 86 and 93-96), and chalcogenides, ${ }^{97,98}$ and they are also located far above the glass-transition temperature, as in the case of water. Thus, we argue that these behaviors may also be caused by local structural orderings and may not be related to the glass transition. This may be reasonable since many of these liquids have a tendency to form local structures ${ }^{30}$ such as tetrahedral or icosahedral structures. ${ }^{99,100}$

The two-state feature provides water with a unique degree of freedom, the fraction of locally favored structures. This makes water unique from ordinary liquids: water can change physical and chemical properties in a flexible manner, responding to the change of various conditions such as temperature, pressure, other external fields, and the addition of solutes or ions. This flexibility of water plays a crucial role in biological and geological situations.

\section{SUPPLEMENTARY MATERIAL}

See supplementary material for movie depicting Fig. 7. The movie shows the local structure fluctuation around a randomly chosen water molecule during 123 ps $\left(\cong 0.05 \tau_{2}\right)$ at 1 bar and $250 \mathrm{~K}$.

\section{ACKNOWLEDGMENTS}

This study was partly supported by Scientific Research (S)\&(A) and Specially Promoted Research (Grant Nos. 21224011, 18H03675, and 25000002, respectively) from the Japan Society for the Promotion of Science (JSPS). J.R. acknowledges support from the European Research Council Grant DLV-759187 and the Royal Society University Research Fellowship.

\section{APPENDIX A: DYNAMIC HETEROGENEITIES}

Dynamic heterogeneity describing the fluctuations of dynamics over time and space is measured by four-point correlation functions. ${ }^{101}$ A four-point susceptibility $\chi_{4}^{\mathrm{T}, \mathrm{R}}(t)$ is defined to characterize the degree of dynamic heterogeneity,

$$
\chi_{4}^{\mathrm{T}, \mathrm{R}}(t)=\frac{1}{N}\left[\left\langle Q(t)^{2}\right\rangle-\langle Q(t)\rangle^{2}\right]
$$

where $Q(t)$ is an overlap function that measures the degree of overlap between two configurations,

$$
Q(t)=\sum_{i}^{N} w_{i}^{\mathrm{T}, \mathrm{R}}(t) .
$$

In order to measure the rotational and translational dynamic heterogeneities separately, the overlap functions are defined as $w_{i}^{\mathrm{T}}(t)=\delta\left(r_{\mathrm{c}}-\Delta \boldsymbol{r}_{i}(t)\right)$ and $w_{i}^{\mathrm{R}}(t)=\delta\left(\varphi_{\mathrm{c}}-\Delta \varphi_{i}(t)\right)$, respectively. Here, $\Delta \boldsymbol{r}_{i}(t)$ and $\varphi_{i}(t)$ are the translational displacement of oxygen and rotational angle of dipole moment of water $i$ during time $t$, respectively. $r_{\mathrm{c}}=1 \AA$ and $\varphi_{\mathrm{c}}=30^{\circ}$ are set as threshold for translational and rotational motions,

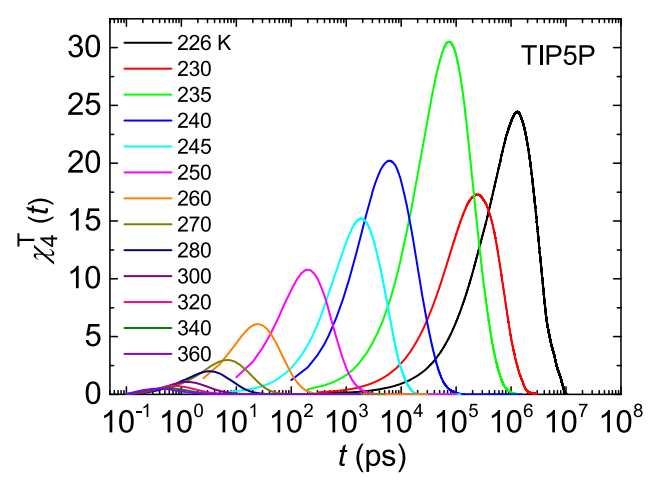

FIG. 22. Translational four-point susceptibility $\chi_{4}^{\mathrm{T}}(t)$ at 1 bar for TIP5P water. $\chi_{4}^{\mathrm{T}}(t)$ maximizes at a dynamic time scale $\tau_{4}$. The curves for 226 , 230, and $235 \mathrm{~K}$ were averaged over 3, 10, and 4 independent trajectories. Reproduced with permission from Fig. S7 of Shi et al., Proc. Natl. Acad. Sci. U. S. A. 115, 9444-9449 (2018). Copyright 2018 Proceedings of the National Academy of Sciences, USA.

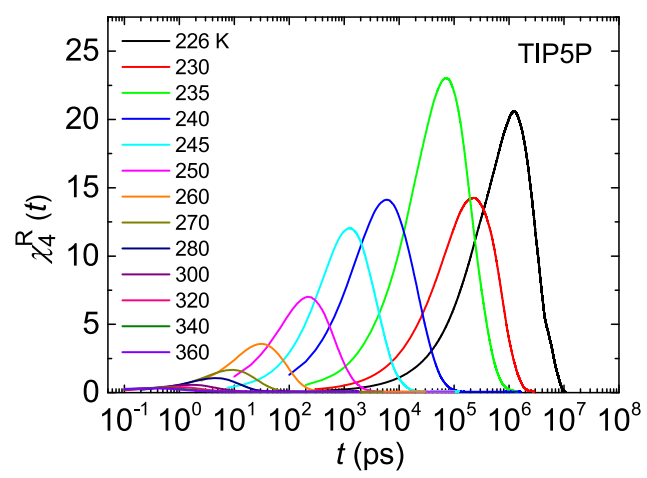

FIG. 23. Rotational four-point susceptibility $\chi_{4}^{\mathrm{R}}(t)$ at 1 bar for TIP5P water. $\chi_{4}^{\mathrm{R}}(t)$ maximizes at a dynamic time scale $\tau_{4}$. The curves for 226, 230, and $235 \mathrm{~K}$ were averaged over 3,10, and 4 independent trajectories. Reproduced with permission from Fig. S8 of Shi et al., Proc. Natl. Acad. Sci. U. S. A. 115, 9444-9449 (2018). Copyright 2018 Proceedings of the National Academy of Sciences, USA.

respectively. Both translational and rotational four-point susceptibilities $\chi_{4}^{\mathrm{T}, \mathrm{R}}(t)$ maximize at the time scale $\tau_{4}$ (Figs. 22 and 23) and the peak heights $\chi_{4}^{\mathrm{T}, \mathrm{R}}\left(\tau_{4}\right)$ as a function of temperature both maximize at the dynamic Schottky line where $s^{\mathrm{D}}=\frac{1}{2}$. This result demonstrates that not only translational but also rotational dynamic heterogeneities maximize at $T_{s^{\mathrm{D}}=\frac{1}{2}}$, confirming the two-state feature of supercooled water.

\section{APPENDIX B: ADDITIONAL RESULTS FOR ST2 WATER}

Figures 24 and 25 show the temperature and pressure dependences of local structures in ST2 water, respectively. The local structures of water were characterized by the structural descriptor $\zeta$, H-bond number, coordination number, and tetrahedral parameter $q$. In agreement with TIP5P water, ST2 water also shows a clear two-state feature, with the $S$ state having higher order, more (four on average) H-bonds, and smaller local density than the $\rho$ state.

Figures 26 and 27 show the translational and rotational four-point susceptibility $\chi_{4}^{\mathrm{T}, \mathrm{R}}(t)$ in ST2 water, respectively. $\chi_{4}^{\mathrm{T}, \mathrm{R}}(t)$ maximizes at a time scale $\tau_{4}$. Similar to TIP5P 


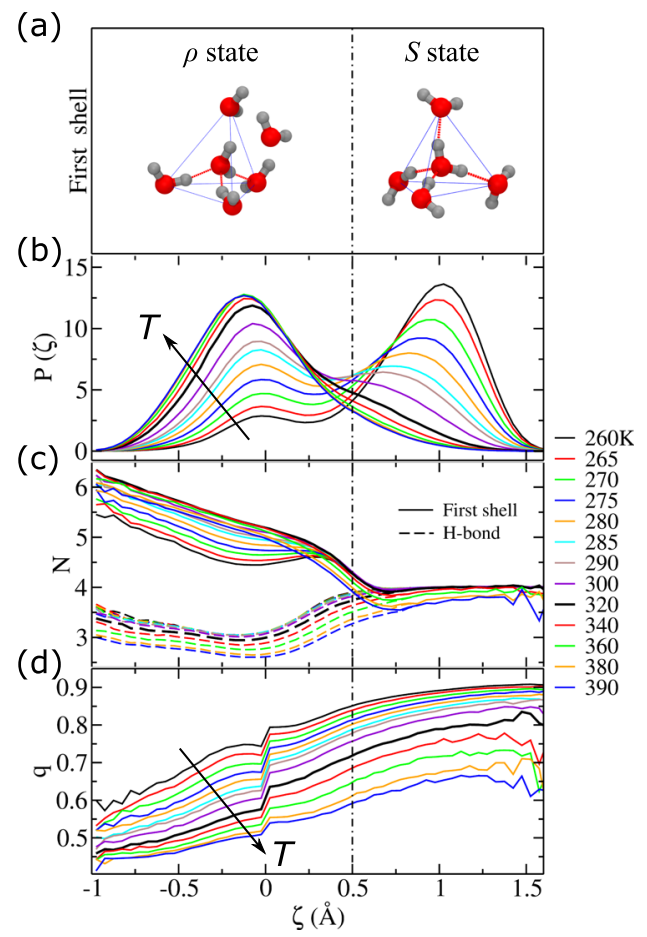

FIG. 24. Local structures of ST2 water at 1 bar for different temperatures. (a) Typical snapshots of water's local structure for static $\rho$ and $S$ states. The dotted red lines represent H-bonds, and solid blue lines show the tetrahedral structure. (b) Distribution of order parameter $\zeta$, which shows a clear bimodal feature. (c) Numbers of first-shell (solid lines) and H-bonded (dashed lines) neighbors per water molecule as a function of $\zeta$. (d) Tetrahedral parameter $q$ as a function of $\zeta$. As illustrated in (a), the $S$ state has higher order, more H-bonds, and smaller local density than the $\rho$ state. The arrows denote the direction of increasing temperature. Panel (a) in this figure is reproduced with permission from Fig. 1 of Shi et al., Proc. Natl. Acad. Sci. U. S. A. 115, 9444-9449 (2018). Copyright 2018 Proceedings of the National Academy of Sciences, USA.

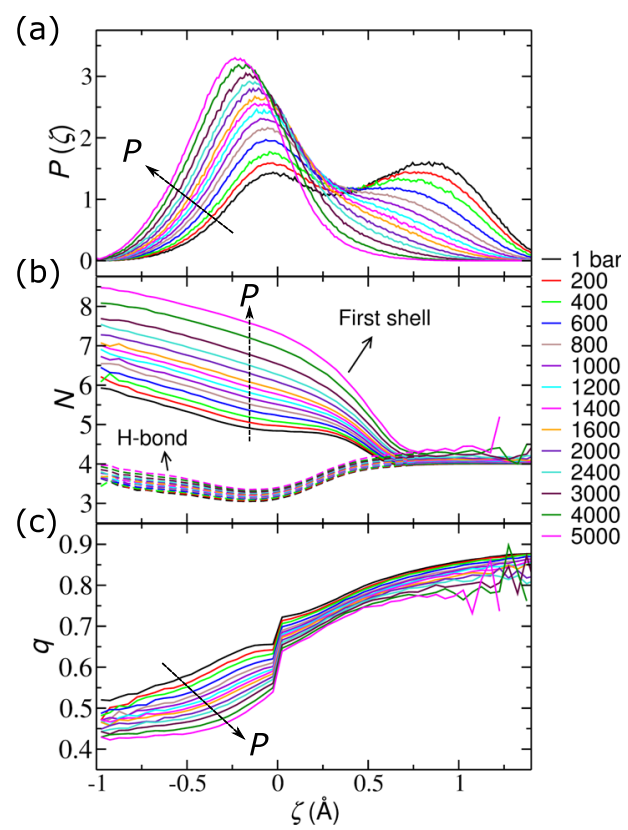

FIG. 25. Local structures of ST2 water at $280 \mathrm{~K}$ for different pressures. (a) Distribution of order parameter $\zeta$. (b) Numbers of first-shell (solid lines) and H-bonded (dashed lines) water as a function of $\zeta$. (c) Tetrahedral parameter $q$ as a function of $\zeta$. Two-state features remain even at high pressures. The dashed arrows denote the direction of increasing pressure.

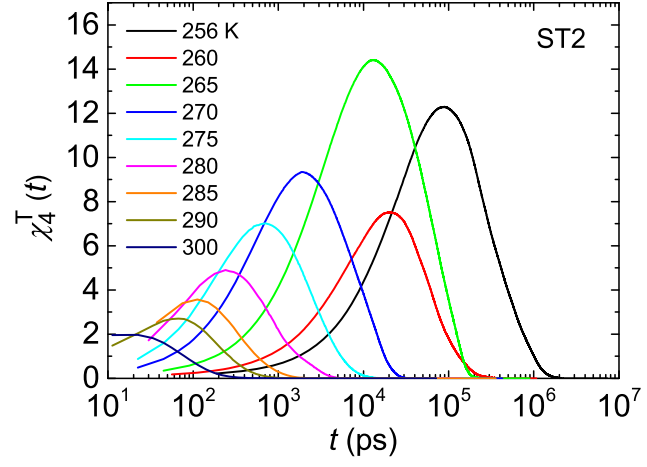

FIG. 26. Translational four-point susceptibility $\chi_{4}^{\mathrm{T}}(t)$ at 1 bar for ST2 water. $\chi_{4}^{\mathrm{T}}(t)$ maximizes at a dynamic time scale $\tau_{4}$. Reproduced with permission from Fig. S9 of Shi et al., Proc. Natl. Acad. Sci. U. S. A. 115, 9444-9449 (2018). Copyright 2018 Proceedings of the National Academy of Sciences, USA.

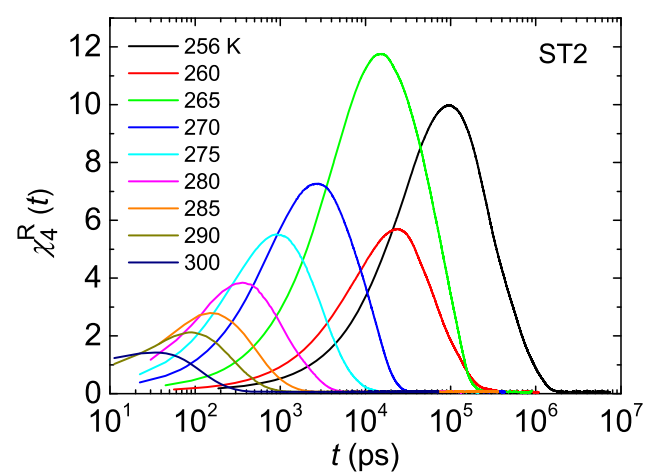

FIG. 27. Rotational four-point susceptibility $\chi_{4}^{\mathrm{R}}(t)$ at 1 bar for ST2 water. $\chi_{4}^{\mathrm{R}}(t)$ maximizes at a dynamic time scale $\tau_{4}$. Reproduced with permission from Fig. S10 of Shi et al., Proc. Natl. Acad. Sci. U. S. A. 115, 9444-9449 (2018). Copyright 2018 Proceedings of the National Academy of Sciences, USA.

water, the peak heights $\chi_{4}^{\mathrm{T}, \mathrm{R}}\left(\tau_{4}\right)$ as a function of temperature maximize at the dynamic Schottky line where $s^{\mathrm{D}}=\frac{1}{2}$, again confirming the two-state feature of supercooled water.

In Fig. 28, we compare the diffusion coefficients of ST2 water calculated in the present work by a hybrid Monte Carlo method, after a simple scaling in time, with those calculated

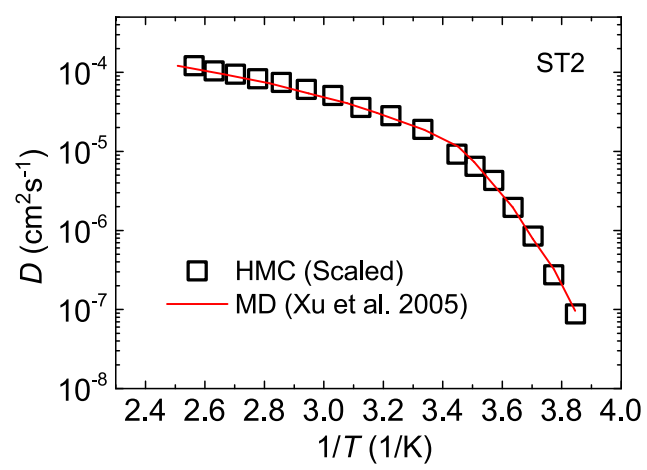

FIG. 28. Diffusion coefficient as a function of inverse temperature in ST2 water at 1 bar. Diffusion coefficients $(D)$ calculated in the present work by a hybrid Monte Carlo method, after a simple scaling in time, agree very well with those calculated by molecular dynamics simulation. ${ }^{8}$ Reproduced with permission from Fig. S14 of Shi et al., Proc. Natl. Acad. Sci. U. S. A. 115, 9444-9449 (2018). Copyright 2018 Proceedings of the National Academy of Sciences, USA. 
by molecular dynamics simulation. ${ }^{8}$ The result shows that the hybrid Monte Carlo method gives the right temperature dependence of water's dynamics. The same time scaling was also applied to Figs. 14, 26, and 27.

${ }^{1}$ C. A. Angell, "Supercooled water," Annu. Rev. Phys. Chem. 34, 593-630 (1983).

${ }^{2}$ O. Mishima and H. E. Stanley, "The relationship between liquid, supercooled and glassy water," Nature 396, 329-335 (1998).

${ }^{3}$ P. G. Debenedetti, "Supercooled and glassy water," J. Phys.: Condens. Matter 15, R1669-R1726 (2003).

${ }^{4}$ A. Nilsson and L. G. M. Pettersson, "The structural origin of anomalous properties of liquid water," Nat. Commun. 6, 8998 (2015).

${ }^{5}$ P. Gallo, K. Amann-Winkel, C. A. Angell, M. A. Anisimov, F. Caupin, C. Chakravarty, E. Lascaris, T. Loerting, A. Z. Panagiotopoulos, J. Russo, J. A. Sellberg, H. E. Stanley, H. Tanaka, C. Vega, L. Xu, and L. G. M. Pettersson, "Water: A tale of two liquids," Chem. Rev. 116, 7463-7500 (2016).

${ }^{6}$ P. H. Handle, T. Loerting, and F. Sciortino, "Supercooled and glassy water: Metastable liquid (s), amorphous solid (s), and a no-man's land," Proc. Natl. Acad. Sci. U. S. A. 114, 13336 (2017).

${ }^{7}$ P. H. Poole, F. Sciortino, U. Essmann, and H. E. Stanley, "Phase behaviour of metastable water," Nature 360, 324-328 (1992).

${ }^{8}$ L. Xu, P. Kumar, S. V. Buldyrev, S. H. Chen, P. H. Poole, F. Sciortino, and H. E. Stanley, "Relation between the Widom line and the dynamic crossover in systems with a liquid-liquid phase transition," Proc. Natl. Acad. Sci. U. S. A. 102, 16558-16562 (2005).

${ }^{9}$ S. Cerveny, F. Mallamace, J. Swenson, M. Vogel, and L. Xu, "Confined water as model of supercooled water," Chem. Rev. 116, 7608-7625 (2016).

${ }^{10}$ J. L. F. Abascal and C. Vega, "Widom line and the liquid-liquid critical point for the tip4p/2005 water model," J. Chem. Phys. 133, 234502 (2010).

${ }^{11}$ J. C. Palmer, F. Martelli, Y. Liu, R. Car, A. Z. Panagiotopoulos, and P. G. Debenedetti, "Metastable liquid-liquid transition in a molecular model of water," Nature 510, 385-388 (2014).

${ }^{12}$ K. H. Kim, A. Späh, H. Pathak, F. Perakis, D. Mariedahl, K. AmannWinkel, J. A. Sellberg, J. H. Lee, S. Kim, J. Park et al., "Maxima in the thermodynamic response and correlation functions of deeply supercooled water," Science 358, 1589-1593 (2017).

${ }^{13}$ S. Woutersen, B. Ensing, M. Hilbers, Z. Zhao, and C. A. Angell, "A liquidliquid transition in supercooled aqueous solution related to the HDA-LDA transition," Science 359, 1127-1131 (2018).

${ }^{14}$ K. E. Bett and J. B. Cappi, "Effect of pressure on the viscosity of water," Nature 207, 620-621 (1965).

${ }^{15} \mathrm{E}$. W. Lang and H. D. Lüdemann, "High pressure $\mathrm{O}^{17}$ longitudinal relaxation time studies in supercooled $\mathrm{H}_{2} \mathrm{O}$ and $\mathrm{D}_{2} \mathrm{O}$," Ber. Bunsenges. Phys. Chem. 85, 603-611 (1981).

${ }^{16}$ R. Torre, P. Bartolini, and R. Righini, "Structural relaxation in supercooled water by time-resolved spectroscopy," Nature 428, 296-299 (2004).

${ }^{17}$ P. Gallo, F. Sciortino, P. Tartaglia, and S.-H. Chen, "Slow dynamics of water molecules in supercooled states," Phys. Rev. Lett. 76, 2730 (1996).

${ }^{18}$ F. Sciortino, P. Gallo, P. Tartaglia, and S.-H. Chen, "Supercooled water and the kinetic glass transition," Phys. Rev. E 54, 6331 (1996).

${ }^{19}$ L. Fabbian, A. Latz, R. Schilling, F. Sciortino, P. Tartaglia, and C. Theis, "Molecular mode-coupling theory for supercooled liquids: Application to water," Phys. Rev. E 60, 5768 (1999).

${ }^{20}$ F. Sciortino and W. Kob, "Debye-waller factor of liquid silica: Theory and simulation," Phys. Rev. Lett. 86, 648 (2001).

${ }^{21}$ K. Ito, C. T. Moynihan, and C. A. Angell, "Thermodynamic determination of fragility in liquids and a fragile-to-strong liquid transition in water," Nature 398, 492-495 (1999).

${ }^{22}$ A. Faraone, L. Liu, C. Y. Mou, C. W. Yen, and S. H. Chen, "Fragile-tostrong liquid transition in deeply supercooled confined water," J. Chem. Phys. 121, 10843-10846 (2004).

${ }^{23}$ C. A. Angelll, "Insights into phases of liquid water from study of its unusual glass-forming properties," Science 319, 582-587 (2008).

${ }^{24}$ Y. Zhang, M. Lagi, E. Fratini, P. Baglioni, E. Mamontov, and S. H. Chen, "Dynamic susceptibility of supercooled water and its relation to the dynamic crossover phenomenon," Phys. Rev. E 79, 040201 (2009).

${ }^{25}$ P. Gallo, M. Rovere, and S.-H. Chen, "Dynamic crossover in supercooled confined water: Understanding bulk properties through confinement," J. Phys. Chem. Lett. 1, 729-733 (2010).
${ }^{26}$ Z. Wang, P. Le, K. Ito, J. B. Leao, M. Tyagi, and S. H. Chen, "Dynamic crossover in deeply cooled water confined in MCM- 41 at 4 kbar and its relation to the liquid-liquid transition hypothesis," J. Chem. Phys. 143, 114508 (2015).

${ }^{27}$ M. De Marzio, G. Camisasca, M. Rovere, and P. Gallo, "Mode coupling theory and fragile to strong transition in supercooled TIP4P/2005 water," J. Chem. Phys. 144, 074503 (2016).

${ }^{28}$ M. De Marzio, G. Camisasca, M. Rovere, and P. Gallo, "Microscopic origin of the fragile to strong crossover in supercooled water: The role of activated processes," J. Chem. Phys. 146, 084502 (2017).

${ }^{29}$ R. Shi, J. Russo, and H. Tanaka, "Origin of the emergent fragile-to-strong transition in supercooled water," Proc. Natl. Acad. Sci. U. S. A. 115, 94449449 (2018).

${ }^{30} \mathrm{H}$. Tanaka, "Bond orientational order in liquids: Towards a unified description of water-like anomalies, liquid-liquid transition, glass transition, and crystallization,” Eur. Phys. J. E 35, 113 (2012).

${ }^{31} \mathrm{H}$. Tanaka, "Simple physical explanation of the unusual thermodynamic behavior of liquid water," Phys. Rev. Lett. 80, 5750 (1998).

${ }^{32}$ H. Tanaka, "Simple physical model of liquid water," J. Chem. Phys. 112, 799-809 (2000).

${ }^{33} \mathrm{H}$. Tanaka, "Thermodynamic anomaly and polyamorphism of water," Europhys. Lett. 50, 340-346 (2000).

${ }^{34} \mathrm{H}$. Tanaka, "A new scenario of the apparent fragile-to-strong transition in tetrahedral liquids: Water as an example," J. Phys.: Condens. Matter 15, L703-L711 (2003).

${ }^{35}$ V. Holten and M. A. Anisimov, "Entropy-driven liquid-liquid separation in supercooled water," Sci. Rep. 2, 713 (2012).

${ }^{36}$ V. Holten, D. T. Limmer, V. Molinero, and M. A. Anisimov, "Nature of the anomalies in the supercooled liquid state of the mw model of water," J. Chem. Phys. 138, 174501 (2013).

${ }^{37}$ V. Holten, J. C. Palmer, P. H. Poole, P. G. Debenedetti, and M. A. Anisimov, "Two-state thermodynamics of the ST2 model for supercooled water," J. Chem. Phys. 140, 104502 (2014).

${ }^{38}$ R. S. Singh, J. W. Biddle, P. G. Debenedetti, and M. A. Anisimov, "Twostate thermodynamics and the possibility of a liquid-liquid phase transition in supercooled TIP4P/2005 water," J. Chem. Phys. 144, 144504 (2016).

${ }^{39}$ J. W. Biddle, R. S. Singh, E. M. Sparano, F. Ricci, M. A. González, C. Valeriani, J. F. Abascal, P. G. Debenedetti, M. A. Anisimov, and F. Caupin, "Two-structure thermodynamics for the TIP4P/2005 model of water covering supercooled and deeply stretched regions," J. Chem. Phys. 146, 034502 (2017).

${ }^{40}$ L. P. Singh, B. Issenmann, and F. Caupin, "Pressure dependence of viscosity in supercooled water and a unified approach for thermodynamic and dynamic anomalies of water," Proc. Natl. Acad. Sci. U. S. A. 114, 4312-4317 (2017).

${ }^{41}$ M. A. Anisimov, M. Duška, F. Caupin, L. E. Amrhein, A. Rosenbaum, and R. J. Sadus, "Thermodynamics of fluid polyamorphism," Phys. Rev. X 8, 011004 (2018).

${ }^{42}$ P. M. de Hijes, E. Sanz, L. Joly, C. Valeriani, and F. Caupin, "Viscosity and self-diffusion of supercooled and stretched water from molecular dynamics simulations," J. Chem. Phys. 149, 094503 (2018).

${ }^{43}$ M. J. Cuthbertson and P. H. Poole, "Mixturelike behavior near a liquidliquid phase transition in simulations of supercooled water," Phys. Rev. Lett. 106, 115706 (2011).

${ }^{44}$ Y. E. Altabet, R. S. Singh, F. H. Stillinger, and P. G. Debenedetti, "Thermodynamic anomalies in stretched water," Langmuir 33, 11771-11778 (2017).

${ }^{45}$ E. Shiratani and M. Sasai, "Molecular scale precursor of the liquid-liquid phase transition of water," J. Chem. Phys. 108, 3264-3276 (1998).

${ }^{46} \mathrm{~J}$. Russo and H. Tanaka, "Understanding water's anomalies with locally favoured structures," Nat. Commun. 5, 3556 (2014).

${ }^{47}$ R. Shi and H. Tanaka, "Microscopic structural descriptor of liquid water," J. Chem. Phys. 148, 124503 (2018).

${ }^{48} \mathrm{R}$. Shi and H. Tanaka, "Impact of local symmetry breaking on the physical properties of tetrahedral liquids," Proc. Natl. Acad. Sci. U. S. A. 115, 1980-1985 (2018)

${ }^{49} \mathrm{H}$. Tanaka, "Two-order-parameter description of liquids: Critical phenomena and phase separation of supercooled liquids," J. Phys.: Condens. Matter 11, L159 (1999).

${ }^{50} \mathrm{H}$. Tanaka, "General view of a liquid-liquid phase transition," Phys. Rev. E 62, 6968 (2000).

${ }^{51}$ M. Yamada, S. Mossa, H. E. Stanley, and F. Sciortino, "Interplay between time-temperature-transformation and the liquid-liquid phase transition in water," Phys. Rev. Lett. 88, 195701 (2002). 
${ }^{52}$ B. Hess, C. Kutzner, D. van der Spoel, and E. Lindahl, "GROMACS 4: Algorithms for highly efficient, load-balanced, and scalable molecular simulation," J. Chem. Theory Comput. 4, 435-447 (2008).

${ }^{53} \mathrm{M}$. W. Mahoney and W. L. Jorgensen, "A five-site model for liquid water and the reproduction of the density anomaly by rigid, nonpolarizable potential functions," J. Chem. Phys. 112, 8910-8922 (2000).

${ }^{54}$ F. H. Stillinger, "Improved simulation of liquid water by molecular dynamics," J. Chem. Phys. 60, 1545-1557 (1974).

${ }^{55}$ A. K. Soper and M. A. Ricci, "Structures of high-density and low-density water," Phys. Rev. Lett. 84, 2881-2884 (2000).

${ }^{56}$ Z. Yan, S. V. Buldyrev, P. Kumar, N. Giovambattista, P. G. Debenedetti, and H. E. Stanley, "Structure of the first-and second-neighbor shells of simulated water: Quantitative relation to translational and orientational order," Phys. Rev. E 76, 051201 (2007).

${ }^{57}$ A. Luzar and D. Chandler, "Effect of environment on hydrogen bond dynamics in liquid water," Phys. Rev. Lett. 76, 928-931 (1996).

${ }^{58}$ A. Luzar and D. Chandler, "Hydrogen-bond kinetics in liquid water," Nature 379, 55 (1996).

${ }^{59}$ J. R. Errington and P. G. Debenedetti, "Relationship between structural order and the anomalies of liquid water," Nature 409, 318-321 (2001).

${ }^{60}$ P.-L. Chau and A. J. Hardwick, "A new order parameter for tetrahedral configurations," Mol. Phys. 93, 511-518 (1998).

${ }^{61} \mathrm{G}$. Walrafen, "Raman spectral studies of water structure," J. Chem. Phys. 40, 3249-3256 (1964).

${ }^{62} \mathrm{G}$. Walrafen, "Raman spectral studies of the effects of temperature on water structure," J. Chem. Phys. 47, 114-126 (1967).

${ }^{63}$ G. Walrafen, M. Fisher, M. Hokmabadi, and W.-H. Yang, "Temperature dependence of the low-and high-frequency Raman scattering from liquid water," J. Chem. Phys. 85, 6970-6982 (1986).

${ }^{64}$ S. Woutersen, U. Emmerichs, and H. Bakker, "Femtosecond mid-IR pump-probe spectroscopy of liquid water: Evidence for a two-component structure," Science 278, 658-660 (1997).

${ }^{65}$ A. Taschin, P. Bartolini, R. Eramo, R. Righini, and R. Torre, "Evidence of two distinct local structures of water from ambient to supercooled conditions," Nat. Commmun. 4, 2401 (2013).

${ }^{66}$ J. A. Sellberg, S. Kaya, V. H. Segtnan, C. Chen, T. Tyliszczak, H. Ogasawara, D. Nordlund, L. G. Pettersson, and A. Nilsson, "Comparison of x-ray absorption spectra between water and ice: New ice data with low pre-edge absorption cross-section," J. Chem. Phys. 141, 034507 (2014).

${ }^{67}$ C. Huang, K. T. Wikfeldt, T. Tokushima, D. Nordlund, Y. Harada, U. Bergmann, M. Niebuhr, T. Weiss, Y. Horikawa, M. Leetmaa et al., "The inhomogeneous structure of water at ambient conditions," Proc. Natl. Acad. Sci. U. S. A. 106, 15214-15218 (2009).

${ }^{68} \mathrm{~J}$. Russo and H. Tanaka, "Assessing the role of static length scales behind glassy dynamics in polydisperse hard disks," Proc. Natl. Acad. Sci. U. S. A. 112, 6920-6924 (2015).

${ }^{69}$ F. Sciortino and S. Fornili, "Hydrogen bond cooperativity in simulated water: Time dependence analysis of pair interactions," J. Chem. Phys. 90, 2786-2792 (1989).

${ }^{70} \mathrm{D}$. Laage and J. T. Hynes, "A molecular jump mechanism of water reorientation," Science 311, 832-835 (2006).

${ }^{71}$ F. Sciortino, A. Geiger, and H. E. Stanley, "Effect of defects on molecular mobility in liquid water," Nature 354, 218 (1991).

${ }^{72}$ R. J. Speedy and C. A. Angell, "Isothermal compressibility of supercooled water and evidence for a thermodynamic singularity at $-45^{\circ} \mathrm{C}$," J. Chem. Phys. 65, 851 (1976).

${ }^{73}$ A. Dehaoui, B. Issenmann, and F. Caupin, "Viscosity of deeply supercooled water and its coupling to molecular diffusion," Proc. Natl. Acad. Sci. U. S. A. 112, 12020-12025 (2015).

${ }^{74} \mathrm{P}$. Gallo and M. Rovere, "Mode coupling and fragile to strong transition in supercooled TIP4P water," J. Chem. Phys. 137, 164503 (2012).

${ }^{75}$ Y. Ni, N. J. Hestand, and J. Skinner, "Diffusion constant in supercooled water as the Widom line is crossed in no man's land," J. Chem. Phys. 148, 191102 (2018).

${ }^{76}$ F. Mallamace, C. Corsaro, P. Baglioni, E. Fratini, and S.-H. Chen, "The dynamical crossover phenomenon in bulk water, confined water and protein hydration water," J. Phys.: Condens. Matter 24, 064103 (2012).

${ }^{77}$ C. Gainaru, A. L. Agapov, V. Fuentes-Landete, K. Amann-Winkel, H. Nelson, K. W. Köster, A. I. Kolesnikov, V. N. Novikov, R. Richert, R. Böhmer, T. Loerting, and A. P. Sokolov, "Anomalously large isotope effect in the glass transition of water," Proc. Natl. Acad. Sci. U. S. A. 111, 17402-17407 (2014).
${ }^{78}$ T. Loerting, V. Fuentes-Landete, P. H. Handle, M. Seidl, K. AmannWinkel, C. Gainaru, and R. Böhmer, "The glass transition in high-density amorphous ice," J. Non-Cryst. Solids 407, 423-430 (2015).

${ }^{79}$ C. Angell, "Water II is a 'strong' liquid," J. Phys. Chem. 97, 6339-6341 (1993).

${ }^{80} \mathrm{C}$. Angell, "Liquid fragility and the glass transition in water and aqueous solutions," Chem. Rev. 102, 2627-2650 (2002).

${ }^{81}$ W. S. Price, H. Ide, and Y. Arata, "Self-diffusion of supercooled water to 238 K using PGSE NMR diffusion measurements,” J. Phys. Chem. A 103, 448-450 (1999).

${ }^{82}$ Y. Xu, N. G. Petrik, R. S. Smith, B. D. Kay, and G. A. Kimmel, "Growth rate of crystalline ice and the diffusivity of supercooled water from 126 to 262 k," Proc. Natl. Acad. Sci. U. S. A. 113, 14921 (2016).

${ }^{83}$ G. P. Johari, A. Hallbrucker, and E. Mayer, "The glass-liquid transition of hyperquenched water," Nature 330, 552-553 (1987).

${ }^{84} \mathrm{~J}$. Horbach and W. Kob, "Static and dynamic properties of a viscous silica melt," Phys. Rev. B 60, 3169 (1999).

${ }^{85}$ I. Saika-Voivod, P. H. Poole, and F. Sciortino, "Fragile-to-strong transition and polyamorphism in the energy landscape of liquid silica," Nature 412, 514-517 (2001).

${ }^{86} \mathrm{C}$. Zhang, L. Hu, Y. Yue, and J. C. Mauro, "Fragile-to-strong transition in metallic glass-forming liquids," J. Chem. Phys. 133, 014508 (2010).

${ }^{87}$ V. Holten, J. V. Sengers, and M. A. Anisimov, "Equation of state for supercooled water at pressures up to $400 \mathrm{MPa}$," J. Phys. Chem. Ref. Data 43, 043101 (2014).

${ }^{88}$ J. Sellberg, C. Huang, T. McQueen, N. Loh, H. Laksmono, D. Schlesinger, R. Sierra, D. Nordlund, C. Hampton, D. Starodub, D. DePonte, M. Beye, C. Chen, A. Martin, A. Barty, K. Wikfeldt, T. Weiss, C. Caronna, J. Feldkamp, L. Skinner, M. Seibert, M. Messerschmidt, G. Williams, S. Boutet, L. Pettersson, M. Bogan, and A. Nilsson, "Ultrafast X-ray probing of water structure below the homogeneous ice nucleation temperature," Nature 510, 381-384 (2014).

${ }^{89} \mathrm{Y}$. Ni and J. Skinner, "Evidence for a liquid-liquid critical point in supercooled water within the E3B3 model and a possible interpretation of the kink in the homogeneous nucleation line," J. Chem. Phys. 144, 214501 (2016).

${ }^{90} \mathrm{Y}$. Ni and J. Skinner, "IR spectra of water droplets in no man's land and the location of the liquid-liquid critical point," J. Chem. Phys. 145, 124509 (2016).

${ }^{91}$ L. Berthier, G. Biroli, J.-P. Bouchaud, W. Kob, K. Miyazaki, and D. Reichman, "Spontaneous and induced dynamic fluctuations in glass formers. I. General results and dependence on ensemble and dynamics," J. Chem. Phys. 126, 184503 (2007).

${ }^{92}$ N. Lačević, F. W. Starr, T. Schrøder, V. Novikov, and S. Glotzer, "Growing correlation length on cooling below the onset of caging in a simulated glass-forming liquid," Phys. Rev. E 66, 030101 (2002).

${ }^{93}$ K. Lad, N. Jakse, and A. Pasturel, "Signatures of fragile-to-strong transition in a binary metallic glass-forming liquid," J. Chem. Phys. 136, 104509 (2012).

${ }^{94}$ Z. Evenson, T. Schmitt, M. Nicola, I. Gallino, and R. Busch, "High temperature melt viscosity and fragile to strong transition in $\mathrm{Zr}-\mathrm{Cu}-\mathrm{Ni}-\mathrm{Al}-\mathrm{Nb}$ (Ti) and $\mathrm{Cu}_{47} \mathrm{Ti}_{34} \mathrm{Zr}_{11} \mathrm{Ni}_{8}$ bulk metallic glasses," Acta Mater. 60, 4712-4719 (2012).

${ }^{95}$ Q. Sun, C. Zhou, Y. Yue, and L. Hu, "A direct link between the fragileto-strong transition and relaxation in supercooled liquids," J. Phys. Chem. Lett. 5, 1170-1174 (2014).

${ }^{96}$ C. Zhou, L. Hu, Q. Sun, H. Zheng, C. Zhang, and Y. Yue, "Structural evolution during fragile-to-strong transition in $\mathrm{CuZr}$ (Al) glass-forming liquids," J. Chem. Phys. 142, 064508 (2015).

${ }^{97}$ J. Orava, D. W. Hewak, and A. L. Greer, "Fragile-to-strong crossover in supercooled liquid Ag-In-Sb-Te studied by ultrafast calorimetry," Adv. Funct. Mater. 25, 4851-4858 (2015).

${ }^{98}$ S. Wei, P. Lucas, and C. A. Angell, "Phase change alloy viscosities down to tg using Adam-Gibbs-equation fittings to excess entropy data: A fragileto-strong transition," J. Appl. Phys. 118, 034903 (2015).

${ }^{99} \mathrm{H}$. Tanaka, "Roles of local icosahedral chemical ordering in glass and quasicrystal formation in metallic glass formers," J. Phys.: Condens. Matter 15, L491 (2003).

${ }^{100} \mathrm{H}$. Tanaka, "Relationship among glass-forming ability, fragility, and shortrange bond ordering of liquids," J. Non-Cryst. Solids 351, 678-690 (2005).

${ }^{101}$ C. Bennemann, C. Donati, J. Baschnagel, and S. C. Glotzer, "Growing range of correlated motion in a polymer melt on cooling towards the glass transition," Nature 399, 246-249 (1999). 